No. 1005

M/G/1 Queue with Multiple Working V acations

by

De- An Wu and Hideaki Takagi

A ugust 2002 


\title{
M/G/1 Queue with Multiple Working Vacations
}

\author{
De-An Wu \\ Doctoral Program in Policy and Planning Sciences, University of Tsukuba \\ 1-1-1 Tennoudai, Tsukuba-shi, Ibaraki 305-8573, Japan \\ phone: +81-298-53-5167; fax: $+81-298-53-5167$ \\ e-mail: tbu@sk.tsukuba.ac.jp \\ and \\ Hideaki Takagi \\ Vice President, University of Tsukuba \\ 1-1-1 Tennoudai, Tsukuba-shi, Ibaraki 305-8577, Japan \\ phone: +81-298-53-2005; fax: +81-298-53-6310 \\ e-mail: takagi@sk.tsukuba.ac.jp
}

\begin{abstract}
We study an $\mathrm{M} / \mathrm{G} / 1$ queue with multiple vacations and exhaustive service discipline such that the server works with different service times rather than completely stops service during a vacation. It is assumed that both service times in a vacation and in a service period are generally distributed random variables. The length of a vacation is also generally distributed. We derive the distributions for the queue size and the system time for an arbitrary customer in the steady state. Several special cases, namely, exponentially distributed vacation lengths and/or exponentially distributed service times in a vacation, are considered. Numerical examples are also presented.
\end{abstract}

\section{Introduction}

We consider an $\mathrm{M} / \mathrm{G} / 1$ queue with multiple vacations such that the server works, say at a low rate, rather than completely stops service during a vacation. Such a vacation is called a working vacation (WV) [8]. The server begins a working vacation of random length following a general distribution at the instance when the queue becomes empty. When a vacation ends, if there are customers in the queue, the server changes to another service rate, say a fast service rate, and the service interrupted at the end of the vacation restarts from the beginning; otherwise, another vacation is taken. The time interval between two successive vacations is called a service period [10, p.107]. The length of a service period is zero if there are no customers waiting in the queue at the end of a vacation. The service times in the service period form an i.i.d. sequence of random variables having a general distribution function. The service times in the vacation are also i.i.d. with a general distribution function. The arrivals of customers occur according to a Poisson process. This queueing system is referred to as an $\mathrm{M} / \mathrm{G} / 1 / \mathrm{WV}$ queue in this paper.

It is well-known that the queueing system with server vacations is useful to model a system in which the server has additional task during a vacation. Thus it has wide applicability in analyzing the performance of computer systems, data communication networks, and production systems. During the last two decades, the queueing systems 
with vacations have been studied extensively. The readers are referred to the surveys of Doshi $[2,3]$ and the monograph of Takagi [10] as well as the references therein. In these studies, it is naturally assumed that the server stops service during the vacation. Recently, Servi and Finn [8] have analyzed an $M / M / 1 / W V$ queue in which the server works at a different rate rather than completely stops service during the vacation. The motivation of their study is that the $\mathrm{M} / \mathrm{M} / 1 / \mathrm{WV}$ queue can be used to approximate a multi-queue system whose service rate is one of two service speeds such that the fast speed mode cyclically moves from queue to queue with exhaustive service. They try to apply the $\mathrm{M} / \mathrm{M} / 1 / \mathrm{WV}$ queue to model a WDM optical access network using multiple wavelengths which can be reconfigured. The latter problem is addressed in [7] specifically. In their model, all random variables, i.e., interarrival times, service times in the service period, service times in the vacation, and the length of a vacation, are exponentially distributed. Therefore, it seems difficult to approximate the multi-queue system precisely.

In this paper, we extend Servi and Finn's M/M/1/WV model to an M/G/1/WV model. We assume that both service times in the service period and in the working vacation as well as the length of a working vacation are generally distributed respectively. This model may be more suitable for approximating the multi-queue system. We recall that Kuczura [6] studies the theory of piecewise Markov process. The piecewise Markov process is a discrete-state, continuous-parameter stochastic process which is Markovian within each contiguous time segment. The method for dealing with an $\mathrm{M} / \mathrm{G} / 1 / \mathrm{WV}$ queue in this paper is the theory of piecewise Markov process.

The rest of this paper is organized as follows. In Section 2 we describe the system and define the notation. Section 3 reviews some results about the transient behavior for an $\mathrm{M} / \mathrm{G} / 1$ queue as a preliminary. In Section 4, an imbedded Markov chain that describes the queue size process in an $\mathrm{M} / \mathrm{G} / 1 / \mathrm{WV}$ queue is introduced, and the probability generating function (PGF) for the steady-state queue size is derived. Based on this, the PGF for the steady-state queue size at an arbitrary time is obtained in Section 5. The system time distribution for an arbitrary customer is analyzed in Section 6. Section 7 examines a special case in which the length of a vacation follows an exponential distribution. Another special situation in which the service time in a vacation is exponentially distributed is considered in Section 8. Numerical examples are also presented in Sections 7 and 8. A summary is given in Section 9.

\section{System Description and Notation}

In our $\mathrm{M} / \mathrm{G} / 1 / \mathrm{WV}$ queue, the server begins a working vacation each time the queue becomes empty, and the length of a working vacation is generally distributed. The service time in a working vacation follows a general distribution. If the server returns from a working vacation to find no customers waiting, it begins another working vacation. If the server returns from a working vacation to find the queue not empty, it switches to a service period in which the service time follows a different distribution.

In Figure 1, we illustrate the queue size and the service process in the $\mathrm{M} / \mathrm{G} / 1 / \mathrm{WV}$ queue. It is noted that the service interrupted at the end of a vacation is lost and it is 


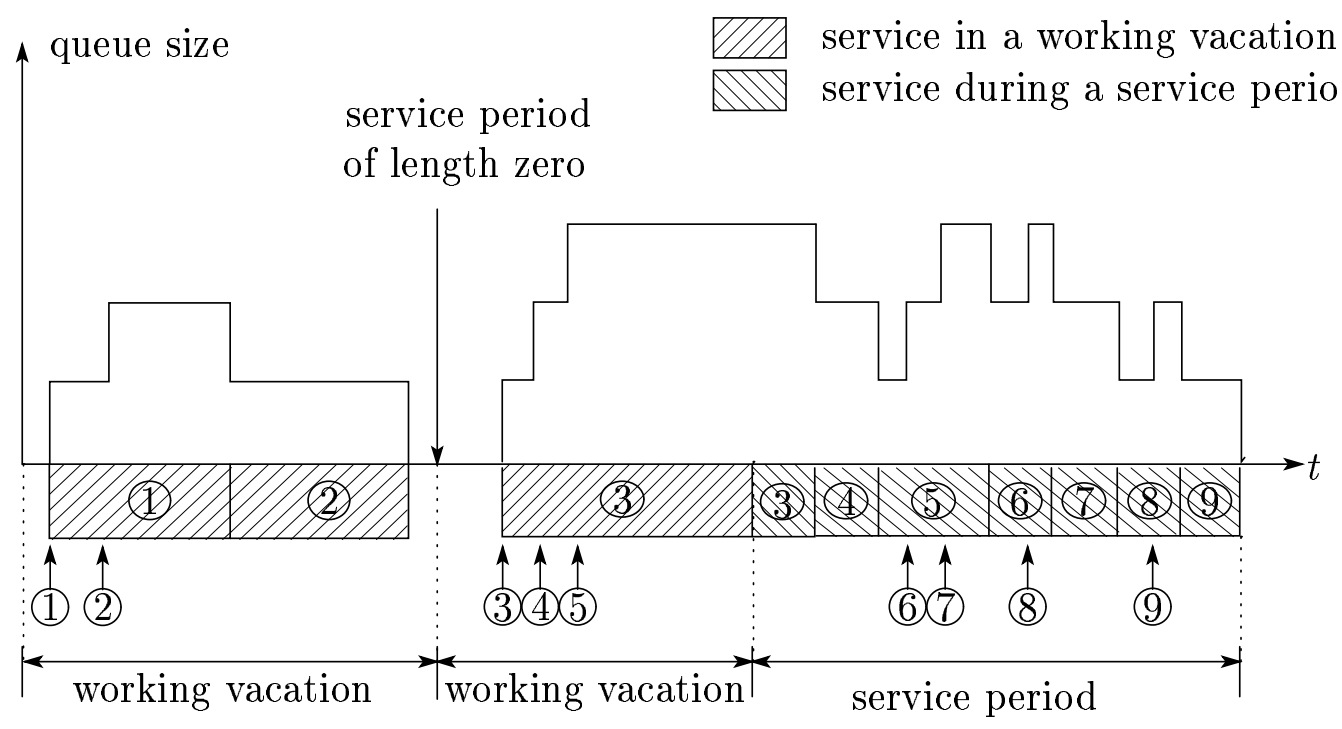

Figure 1: Queue size and service process in an $M / G / 1 / W V$ queueing system.

restarted with a different distribution at the beginning of the following service period.

For the analysis of an $\mathrm{M} / \mathrm{G} / 1 / \mathrm{WV}$ queue, we introduce the following notation:

$\lambda$ arrival rate

$V(t) \quad$ distribution function for the length of a working vacation

$H(t)$ distribution function for the service time in a working vacation

$B(t) \quad$ distribution function for the service during a service period

$\phi(s) \quad$ Laplace-Stieltjes transform (LST) of $V(t)$

$\psi(s) \quad$ LST of $H(t)$

$\beta(s) \quad$ LST of $B(t)$

$v$ mean of $V(t)$

$v_{i} \quad i$ th moment of $V(t)(i=2,3, \ldots)$

$h$ mean of $H(t)$

$b$ mean of $B(t)$

$b_{i} \quad i$ th moment of $B(t)(i=2,3, \ldots)$

\section{Transient Behavior of an M/G/1 Queue}

Before starting our analysis, we review some results about the transient behavior for an $\mathrm{M} / \mathrm{G} / 1$ queue, which will be used in studying the queue size process during a working vacation in an $\mathrm{M} / \mathrm{G} / 1 / \mathrm{WV}$ queue. Therefore we use the following notation: $\lambda$ for the arrival rate, $H(t)$ for the distribution function for the service time, and $\psi(s)$ for the LST of $H(t)$. Let $Y(t)$ be the number of customers (queue size) in the M/G/1 queue at time $t$. Suppose that there is a departure at time $t=-0$ and $Y(0)=i(\geq 0)$. We define the 
transition probability

$$
P_{i, j}(t):=P\{Y(t)=j \mid Y(0)=i\} ; \quad t \geq 0
$$

and its PGF

$$
\Gamma_{i}(z, t):=\sum_{j=0}^{\infty} P_{i, j}(t) z^{j}
$$

The Laplace transform (LT) of $\Gamma_{i}(z, t)$

$$
\gamma_{i}(z, s):=\int_{0}^{\infty} e^{-s t} \Gamma_{i}(z, t) d t ; \quad i=0,1,2, \ldots
$$

is given by $[9$, p.74, eq. (77); 10, p.88, eq. (7.73)]

$$
\gamma_{i}(z, s)=\frac{z^{i+1}[1-\psi(s+\lambda-\lambda z)]+(z-1)(s+\lambda-\lambda z) \psi(s+\lambda-\lambda z) P_{i, 0}^{*}(s)}{(s+\lambda-\lambda z)[z-\psi(s+\lambda-\lambda z)]}
$$

where

$$
P_{i, 0}^{*}(s):=\int_{0}^{\infty} e^{-s t} P_{i, 0}(t) d t=\frac{[\tau(s)]^{i}}{s+\lambda-\lambda \tau(s)}
$$

and $\tau(s)$ is the root with the smallest absolute value of the equation

$$
\tau(s)=\psi[s+\lambda-\lambda \tau(s)] .
$$

Let $N_{i}(t)$ denote the expected number of the customers served in the time interval $(0, t]$ with initial queue size $Y(0)=i(\geq 0)$. The LT of $N_{i}(t)$ is given by $[9$, p.78, eq. $(91)]$

$$
N_{i}^{*}(s):=\int_{0}^{\infty} e^{-s t} N_{i}(t) d t=\frac{\psi(s)}{s[1-\psi(s)]}\left(1-\frac{s[\tau(s)]^{i}}{s+\lambda-\lambda \tau(s)}\right) ; \quad i=0,1,2, \ldots
$$

\section{Queue Size in an Embedded Markov Chain}

We now analyze the $M / G / 1 / W V$ queueing system described in Section 2. The queue size $X(t)$ is the number of customers, including those waiting and in service, in the system at time $t$. Notice that during a working vacation, the queue size process $X(t)$ behaves exactly like the queue size process in an M/G/1 queue described in Section 3. This fact suggests that the queue size during each vacation in an $M / G / 1 / W V$ queue can be analyzed by utilizing the transient solution for the queue size in an $\mathrm{M} / \mathrm{G} / 1$ queue.

Let us consider a discrete-time Markov chain $\left\{X^{(n)} ; n=0,1,2, \ldots\right\}$, where $X^{(n)}$ denotes the queue size immediately after the epoch at which either a vacation or a service starts. For the time-homogeneous Markov chain $\left\{X^{(n)} ; n=0,1,2, \ldots\right\}$, the state transition probability is defined by

$$
p_{i j}=P\left\{X^{(n+1)}=j \mid X^{(n)}=i\right\} ; \quad i, j=0,1,2, \ldots
$$


Because $X^{(n)}=0$ means that the $n$th Markov point is the beginning of a vacation, we have

$$
p_{0 j}=\int_{0}^{\infty} P_{0, j}(t) d V(t) ; \quad j=0,1,2, \ldots
$$

If $X^{(n)}>0$, we must have $X^{(n+1)} \geq X^{(n)}-1$, for the $n$th Markov point is observed at the beginning of a service. Therefore we have

$$
p_{i j}=\left\{\begin{array}{ll}
\int_{0}^{\infty} \frac{(\lambda t)^{j+1-i}}{(j+1-i) !} e^{-\lambda t} d B(t) ; & j \geq i-1 \\
0 ; & j<i-1
\end{array} \quad i=0,1,2, \ldots .\right.
$$

Assuming that this Markov chain is ergodic, the limiting distribution

$$
\pi_{i}:=\lim _{n \rightarrow \infty} P\left\{X^{(n)}=i\right\} ; \quad i=0,1,2, \ldots
$$

satisfies the balance equations

$$
\begin{array}{r}
\pi_{j}=\pi_{0} \int_{0}^{\infty} P_{0, j}(t) d V(t)+\sum_{i=1}^{j+1} \pi_{i} \int_{0}^{\infty} \frac{(\lambda t)^{j+1-i}}{(j+1-i) !} e^{-\lambda t} d B(t) \\
j=0,1,2, \ldots
\end{array}
$$

and normalization condition

$$
\sum_{i=0}^{\infty} \pi_{i}=1
$$

Let us introduce the PGF for $\left\{\pi_{i} ; i=0,1,2, \ldots\right\}$ by

$$
\Pi(z):=\sum_{i=0}^{\infty} \pi_{i} z^{i}
$$

Multiplying (7) by $z^{j}$ and summing over $j=0,1,2, \ldots$, we get

$$
\Pi(z)=\frac{\pi_{0}[z \Psi(z)-\beta(\lambda-\lambda z)]}{z-\beta(\lambda-\lambda z)},
$$

where

$$
\Psi(z):=\int_{0}^{\infty} \Gamma_{0}(z, t) d V(t)
$$

is the PGF for the queue size at the end of a vacation and $\Gamma_{0}(z, t)$ is defined in $(1)$.

Let us transform the real integral (9) appearing in (8) into a complex integral involving $\gamma_{0}(z, s)$ given in $(2)$ and $\phi(s)$, the LST of $V(t)$. To do so, note the inverse transform

$$
\Gamma_{0}(z, t)=\frac{1}{2 \pi \mathbf{i}} \int_{c-\mathbf{i} \infty}^{c+\mathbf{i} \infty} e^{s t} \gamma_{0}(z, s) d s
$$


where $c>0, \mathbf{i}:=\sqrt{-1}$, and the integration $\int_{c-\mathbf{i} \infty}^{c+\mathbf{i} \infty}$ is the Bromwich integral, being written as $\int_{B r}$ hereafter. Furthermore, we have

$$
\int_{0}^{\infty} e^{s t} d V(t)=\phi(-s)
$$

Thus we get

$$
\Psi(z)=\frac{1}{2 \pi \mathbf{i}} \int_{B r} \gamma_{0}(z, s) \phi(-s) d s
$$

Substituting (2) into (11) yields

$$
\Psi(z)=\frac{1}{2 \pi \mathbf{i}} \int_{B r}\left[\frac{z[1-\psi(s+\lambda-\lambda z)]+(z-1) \psi(s+\lambda-\lambda z) \frac{s+\lambda-\lambda z}{s+\lambda-\lambda \tau(s)}}{(s+\lambda-\lambda z)[z-\psi(s+\lambda-\lambda z)]}\right] \phi(-s) d s .
$$

Following Kuczura [5], we may comment on the Bromwich integral in (12) as follows. Since $P_{0, j}(t)$ is the probability, the $\operatorname{LST} \gamma_{0}(z, s)$ of $\Gamma_{0}(z, t)$ is analytic for $|z| \leq 1$ and $\Re(s)>0$. Hence the bracketed part of the integrand in (12) is analytic for $|z| \leq 1$ and $\Re(s)>0$. On the other hand, since $V(t)$ is the distribution function, $\phi(s)$ is analytic for $\Re(s)>0$. For $\Re(s)<0, \phi(s)$ may or may not be analytic. However, $\phi(s)$ is meromorphic for $\Re(s)<0$ in many cases, including the cases in which the distribution function $V(t)$ is exponential, Erlang, and a linear combination thereof.

If we assume that $\phi(s)$ is meromorphic for the left-half plane $\Re(s)<0$, all the poles of $\phi(-s)$ are in the right-half plane $\Re(s)>0$. Hence the integrand in (12) is meromorphic in the right-half plane. Thus we can use the residue theorem to evaluate the integrand over the contour consisting of the line $(c+\mathbf{i} R, c-\mathbf{i} R)$ and a semicircle of radius $R$ in the right-half plane which connects $c-\mathbf{i} R$ with $c+\mathbf{i} R$ counterclockwise. We can choose $c$ and $R$ such that all the poles of $\phi(-s)$ are interior to this contour. Then the Bromwich integrals in (12) are evaluated only at the poles of $\phi(-s)$.

The PGF $\Pi(z)$ in (8) is given in terms of the unknown constant $\pi_{0}$. In order to determine $\pi_{0}$, we first derive several relations. Evaluating $\Psi(z)$ in (12) at $z=1$ gives

$$
\Psi(1)=\frac{1}{2 \pi \mathbf{i}} \int_{B r} \frac{\phi(-s)}{s} d s=1
$$

Differentiating the right-hand side of (12) with respect to $z$ and evaluating the result at $z=1$ yields

$$
\begin{aligned}
\left.\frac{d \Psi(z)}{d z}\right|_{z=1} & =\frac{1}{2 \pi \mathbf{i}} \int_{B r}\left[\frac{\lambda}{s^{2}}-\frac{\psi(s)}{s[1-\psi(s)]}\left(1-\frac{s}{s+\lambda-\lambda \tau(s)}\right)\right] \phi(-s) d s \\
& =\int_{0}^{\infty} d V(t) \frac{1}{2 \pi \mathbf{i}} \int_{B r}\left[\frac{\lambda}{s^{2}}-\frac{\psi(s)}{s[1-\psi(s)]}\left(1-\frac{s}{s+\lambda-\lambda \tau(s)}\right)\right] e^{s t} d s \\
& =\int_{0}^{\infty}\left[\lambda t-N_{0}(t)\right] d V(t) \\
& =\lambda v-N,
\end{aligned}
$$


where we have used (4) with $i=0$, and

$$
N:=\int_{0}^{\infty} N_{0}(t) d V(t)=\frac{1}{2 \pi \mathbf{i}} \int_{B r} \frac{\psi(s)}{s[1-\psi(s)]}\left(1-\frac{s}{s+\lambda-\lambda \tau(s)}\right) \phi(-s) d s
$$

is the expected number of customers served in a vacation. The result in (14) means that the expected queue size at the end of a vacation is equal to the expected number of customers arriving in a vacation minus the expected number of customers served in that vacation. Thus we have $\lambda v-N \geq 0$. By excluding the rare case $\lambda v-N=0$, let us assume that $\lambda v-N>0$ throughout the paper.

We may determine the unknown constant $\pi_{0}$ by recognizing that $\Pi(1)=1$. Evaluating the right-hand side of (8) at $z=1$ and using L'Hospital rule along with relations (13) and (14), we obtain

$$
\pi_{0}=\frac{1-\lambda b}{1+\lambda(v-b)-N}
$$

Substituting this back into (8) yields

$$
\Pi(z)=\frac{(1-\lambda b)[z \Psi(z)-\beta(\lambda-\lambda z)]}{[1+\lambda(v-b)-N][z-\beta(\lambda-\lambda z)]},
$$

where $\Psi(z)$ and $N$ are given in (12) and (15), respectively.

\section{Queue Size at an Arbitrary Time}

We next derive the PGF for the queue size in an M/G/1/MV queue at an arbitrary time. It is observed that the server has service periods and vacations alternately and that it is in one of the two conditions at all times. If we consider the number of customers in the $\mathrm{M} / \mathrm{G} / 1 / \mathrm{MV}$ queue as the system state, it follows from the property of the Poisson arrival process and the assumption of exhaustive service that those points in time at which each vacation starts (i.e., at which each service period ends) are the regeneration points of the system.

According to the renewal theory, if we select an epoch $t^{\prime}$ randomly to observe the system, the probability that the server is in a vacation at time $t^{\prime}$ is

$$
p_{V}:=P\left\{t^{\prime} \in \text { vacation }\right\}=\frac{v}{\sigma+v},
$$

where $\sigma$ is the mean of the length of a service period. Similarly, the probability that the server is in a service period at time $t^{\prime}$ is

$$
p_{B}:=P\left\{t^{\prime} \in \text { service period }\right\}=\frac{\sigma}{\sigma+v} .
$$

Let $S(t)$ be the distribution function for the length of a service period in an $\mathrm{M} / \mathrm{G} / 1 / \mathrm{WV}$ queue, and let its LST be

$$
\sigma(s):=\int_{0}^{\infty} e^{-s t} d S(t)
$$


If there are $i(\geq 0)$ customers in the system at the end of a vacation, the following service period behaves exactly like the busy period in an $\mathrm{M} / \mathrm{G} / 1$ queue beginning with $i$ customers. Therefore, if we denote $\tau_{B}(s)$ the LST of the busy period distribution in an $\mathrm{M} / \mathrm{G} / 1$ queue with arrival rate $\lambda$ and a service time distribution $B(t)$, then

$$
\sigma(s)=\sum_{i=0}^{\infty} \int_{0}^{\infty} P_{0, i}(t) d V(t)\left[\tau_{B}(s)\right]^{i}=\int_{0}^{\infty} \Gamma\left(\tau_{B}(s), t\right) d V(t)=\Psi\left(\tau_{B}(s)\right),
$$

where $\tau_{B}(s)$ is the root with the smallest absolute value of the equation

$$
\tau_{B}(s)=\beta\left[s+\lambda-\lambda \tau_{B}(s)\right]
$$

Thus we obtain

$$
\sigma=-\sigma^{\prime}(0)=-\left.\frac{d \Psi\left(\tau_{B}(s)\right)}{d s}\right|_{s=0}=\frac{b}{1-\lambda b}(\lambda v-N)
$$

where we have used (14). Using (21) in (18) and (19) we get

$$
p_{V}=\frac{(1-\lambda b) v}{v-N b}, \quad p_{B}=\frac{(\lambda v-N) b}{v-N b} .
$$

Since $\sigma>0$ and $\lambda v-N>0$, it follows that

$$
1-\lambda b>0
$$

Thus we have

$$
0<(\lambda v-N) b=\lambda b v-N b<v-N b
$$

The condition (23) means that the arrival rate is less than the service rate during a service period. Therefore, it is a stability condition for our system.

By applying the theorem of total probability, the probability that the queue size $X\left(t^{\prime}\right)=j$ at an arbitrary time $t^{\prime}$ is given by

$P\left\{X\left(t^{\prime}\right)=j\right\}=p_{V} P\left\{X\left(t^{\prime}\right)=j \mid t^{\prime} \in\right.$ vacation $\}+\left(1-\delta_{j 0}\right) p_{B} P\left\{X\left(t^{\prime}\right)=j \mid t^{\prime} \in\right.$ service period $\} ;$

$$
j=0,1,2, \ldots
$$

where $\delta_{j 0}$ is Kronecker's delta. If we multiply (24) by $z^{j}$ and sum over $j=0,1,2, \ldots$, we obtain the PGF

$$
\Phi(z)=p_{V} \Phi_{V}(z)+p_{B} \Phi_{B}(z)
$$

for the queue size at an arbitrary time, where

$$
\Phi(z):=\sum_{j=0}^{\infty} P\left\{X\left(t^{\prime}\right)=j\right\} z^{j}
$$




$$
\Phi_{V}(z):=\sum_{j=0}^{\infty} P\left\{X\left(t^{\prime}\right)=j \mid t^{\prime} \in \text { vacation }\right\} z^{j}
$$

and

$$
\Phi_{B}(z):=\sum_{j=1}^{\infty} P\left\{X\left(t^{\prime}\right)=j \mid t^{\prime} \in \operatorname{service} \text { period }\right\} z^{j} .
$$

To derive $\Phi_{V}(z)$, note that the interval between an arbitrary time in a vacation and the starting time of that vacation corresponds to the deficit of the vacation time in the renewal theory. The distribution function for the deficit is given by

$$
\hat{V}(t):=\frac{1}{v} \int_{0}^{t}[1-V(x)] d x,
$$

and its LST is

$$
\hat{\phi}(s):=\int_{0}^{\infty} e^{-s t} d \hat{V}(t)=\frac{1}{v} \int_{0}^{\infty} e^{-s t}[1-V(t)] d t=\frac{1-\phi(s)}{v s} .
$$

Conditioning on the length of the deficit of a vacation time, we have

$$
P\left\{X\left(t^{\prime}\right)=j \mid t^{\prime} \in \text { vacation }\right\}=\int_{0}^{\infty} P_{0, j}(t) d \hat{V}(t) ; \quad j=0,1,2, \ldots
$$

Substituting (29) into (26) and using the relation similar to (10), we obtain

$$
\Phi_{V}(z)=\int_{0}^{\infty} \Gamma_{0}(z, t) d \hat{V}(t)=\hat{\Psi}(z)
$$

where

$$
\hat{\Psi}(z):=\frac{1}{2 \pi \mathbf{i}} \int_{B r}\left[\frac{z[1-\psi(s+\lambda-\lambda z)]+(z-1) \psi(s+\lambda-\lambda z) \frac{s+\lambda-\lambda z}{s+\lambda-\lambda \tau(s)}}{(s+\lambda-\lambda z)[z-\psi(s+\lambda-\lambda z)]}\right] \hat{\phi}(-s) d s
$$

is the PGF for the queue size at an arbitrary time during a vacation. As in (12), the Bromwich integral is evaluated only at the poles of $\hat{\phi}(-s)$ in the right-half plane $\Re(s)>0$ in most cases.

We next determine the PGF $\Phi_{B}(z)$. Conditioning on the number of customers in the system at the epoch at which a service begins, and integrating with deficit of a service time, we obtain

$$
P\left\{X\left(t^{\prime}\right)=j \mid t^{\prime} \in \text { service period }\right\}=\sum_{i=1}^{j} \frac{\pi_{i}}{1-\pi_{0}} \int_{0}^{\infty} \frac{(\lambda t)^{j-i}}{(j-i) !} e^{-\lambda t} d \hat{B}(t) ; \quad j=1,2, \ldots,
$$

where

$$
\hat{B}(t):=\frac{1}{b} \int_{0}^{t}[1-B(x)] d x .
$$


Substituting (32) into (27), rearranging terms, and using (8) and (16) gives

$$
\begin{aligned}
\Phi_{B}(z) & =\frac{\pi_{0}}{1-\pi_{0}}\left(\frac{\Pi(z)}{\pi_{0}}-1\right) \hat{\beta}(\lambda-\lambda z) \\
& =\left(\frac{1-\lambda b}{b(\lambda v-N)}\right)\left(\frac{z[\Psi(z)-1][1-\beta(\lambda-\lambda z)]}{\lambda(1-z)[z-\beta(\lambda-\lambda z)]}\right)
\end{aligned}
$$

where

$$
\hat{\beta}(s):=\int_{0}^{\infty} e^{-s t} d \hat{B}(t)=\frac{1-\beta(s)}{b s} .
$$

Using relations (22), (30), and (33) in (25), we obtain

$$
\Phi(z)=\frac{1-\lambda b}{v-N b}\left(v \hat{\Psi}(z)+\frac{z[\Psi(z)-1][1-\beta(\lambda-\lambda z)]}{\lambda(1-z)[z-\beta(\lambda-\lambda z)]}\right) .
$$

Letting $z=1$ in (34) recovers

$$
\begin{aligned}
\Phi(1) & =\frac{1-\lambda b}{v-N b}\left(v \hat{\Psi}(1)+\lim _{z \rightarrow 1} \frac{z[\Psi(z)-1][1-\beta(\lambda-\lambda z)]}{\lambda(1-z)[z-\beta(\lambda-\lambda z)]}\right) \\
& =\frac{1-\lambda b}{v-N b}\left(v+\frac{\left[\Psi(1)-1+\Psi^{\prime}(1)\right] \lambda b}{\lambda(1-\lambda b)}\right) \\
& =\frac{1-\lambda b}{v-N b}\left(v+\frac{(\lambda v-N) b}{1-\lambda b}\right)=1,
\end{aligned}
$$

where we have used (13), L'Hospital's rule, and

$$
\hat{\Psi}(1)=\int_{0}^{\infty} \Gamma(1, t) \frac{1-V(t)}{v} d t=1 .
$$

Differentiating (34) and using L'Hospital's law four times, we have

$$
\mathrm{E}[X]=\frac{1-\lambda b}{v-N b}\left[v \hat{\Psi}^{\prime}(1)+\frac{\left[\lambda b_{2}+2(1-\lambda b) b\right] \Psi^{\prime}(1)}{2(1-\lambda b)^{2}}+\frac{b \Psi^{\prime \prime}(1)}{2(1-\lambda b)}\right]
$$

where $\Psi^{\prime}(1)$ is given by $(14)$,

$$
\begin{aligned}
\Psi^{\prime \prime}(1)= & \frac{1}{\pi \mathbf{i}} \int_{B r}\left[\frac{\lambda^{2}}{s^{3}}-\frac{\lambda \psi(s)}{s^{2}[1-\psi(s)]}+\frac{\psi(s)+\lambda \psi^{\prime}(s)}{s[1-\psi(s)]^{2}}\right. \\
& \left.\times\left(1-\frac{s}{s+\lambda-\lambda \tau(s)}\right)\right] \phi(-s) d s
\end{aligned}
$$

and

$$
\hat{\Psi}^{\prime}(1)=\frac{1}{2 \pi \mathbf{i}} \int_{B r}\left[\frac{\lambda}{s^{2}}-\frac{\psi(s)}{s[1-\psi(s)]}\left(1-\frac{s}{s+\lambda-\lambda \tau(s)}\right)\right] \hat{\phi}(-s) d s .
$$


Differentiating (34) twice and using L'Hospital's rule six times, we have

$$
\begin{aligned}
\mathrm{E}\left[X^{2}\right]= & \frac{1-\lambda b}{v-N b}\left[v\left[\hat{\Psi}^{\prime}(1)+\hat{\Psi}^{\prime \prime}(1)\right]+\left(\frac{\lambda b_{2}+2(1-\lambda b) b}{2(1-\lambda b)^{2}}\right.\right. \\
& \left.+\frac{\lambda\left[3 \lambda^{2} b_{2}^{2}+2(1-\lambda b)\left(3 b_{2}+\lambda b_{3}\right)\right]}{6(1-\lambda b)^{3}}\right) \Psi^{\prime}(1) \\
& \left.+\left(\frac{b}{2(1-\lambda b)}+\frac{\lambda b_{2}+2(1-\lambda b) b}{2(1-\lambda b)^{2}}\right) \Psi^{\prime \prime}(1)+\frac{b \Psi^{(3)}(1)}{3(1-\lambda b)}\right]
\end{aligned}
$$

where

$$
\begin{aligned}
\Psi^{(3)}(1)= & \frac{3}{2 \pi \mathbf{i}} \int_{B r}\left[\frac{2 \lambda^{3}}{s^{4}}+\frac{2 \lambda\left[\psi(s)+\lambda \psi^{\prime}(s)\right]}{s^{2}[1-\psi(s)]^{2}}-\frac{2 \lambda^{2} \psi(s)}{s^{3}[1-\psi(s)]}\right. \\
& -\left(\frac{2 \psi(s)+2 \lambda \psi^{\prime}(s)\left[1+\psi(s)+\lambda \psi^{\prime}(s)\right]}{s[1-\psi(s)]^{3}}+\frac{\lambda^{2} \psi^{\prime \prime}(s)}{s[1-\psi(s)]^{2}}\right) \\
& \left.\times\left(1-\frac{s}{s+\lambda-\lambda \tau(s)}\right)\right] \phi(-s) d s
\end{aligned}
$$

and

$$
\begin{aligned}
\hat{\Psi}^{\prime \prime}(1)= & \frac{1}{\pi \mathbf{i}} \int_{B r}\left[\frac{\lambda^{2}}{s^{3}}-\frac{\lambda \psi(s)}{s^{2}[1-\psi(s)]}+\frac{\psi(s)+\lambda \psi^{\prime}(s)}{s[1-\psi(s)]^{2}}\right. \\
& \left.\times\left(1-\frac{s}{s+\lambda-\lambda \tau(s)}\right)\right] \hat{\phi}(-s) d s .
\end{aligned}
$$

\section{Remark}

In Appendix A we show that if the server does not work during a working vacation, $\Phi(z)$ in (34) becomes

$$
\Phi(z)=\frac{(1-\lambda b)[1-\phi(\lambda-\lambda z)] \beta(\lambda-\lambda z)}{\lambda v[\beta(\lambda-\lambda z)-z]} .
$$

This is the PGF for the queue size at an arbitrary time in an $M / G / 1$ queue with multiple non-working vacations $[10$, p.124, eq. (2.19b)].

\section{System Time of an Arbitrary Customer}

We proceed to determine the total time $T$ for an arbitrary customer to stay in the system (system time). We define the LST of the distribution function for the system time $T$ by

$$
\alpha(s):=\int_{0}^{\infty} e^{-s t} d P\{T \leq t\}
$$


To derive $\alpha(s)$, we use a theorem in [4] (the distributional form of Little's law), which states as follows:

$$
\Phi(z)=\alpha(\lambda-\lambda z)
$$

if following four conditions hold: (a) the arrivals are Poisson; (b) all arriving customers enter the system, and remain in the system until served; (c) the customers leave the system one at a time in the order of arrivals; and (d) for any time $t$, the arrival process after time $t$ and the time in the system of any customer arriving before $t$ are independent.

Obviously, these four conditions are satisfied for our M/G/1/WV queue. Substituting $z=1-s / \lambda$ in $(34)$ gives

$$
\alpha(s)=\frac{1-\lambda b}{v-N b}\left(v \hat{\Psi}(1-s / \lambda)+\frac{(\lambda-s)[\Psi(1-s / \lambda)-1][1-\beta(s)]}{s\{\lambda[1-\beta(s)]-s\}}\right) .
$$

By taking derivative and using L'Hospital's rule, the mean $E[T]$ and the second moment $\mathrm{E}\left[T^{2}\right]$ of the system time $T$ are given by

$$
\mathrm{E}[T]=\frac{\mathrm{E}[X]}{\lambda}=\frac{1-\lambda b}{(v-N b) \lambda}\left[v \hat{\Psi}^{\prime}(1)+\frac{\left[\lambda b_{2}+2(1-\lambda b) b\right] \Psi^{\prime}(1)}{2(1-\lambda b)^{2}}+\frac{b \Psi^{\prime \prime}(1)}{2(1-\lambda b)}\right]
$$

and

$$
\begin{aligned}
\mathrm{E}\left[T^{2}\right]= & \frac{1-\lambda b}{(v-N b) \lambda^{2}}\left[v \hat{\Psi}^{\prime \prime}(1)+\frac{\lambda\left[3 \lambda^{2} b_{2}^{2}+2(1-\lambda b)\left(3 b_{2}+\lambda b_{3}\right)\right] \Psi^{\prime}(1)}{6(1-\lambda b)^{3}}\right. \\
& \left.+\frac{\left[\lambda b_{2}+2(1-\lambda b) b\right] \Psi^{\prime \prime}(1)}{2(1-\lambda b)^{2}}+\frac{b \Psi^{(3)}(1)}{3(1-\lambda b)}\right] .
\end{aligned}
$$

\section{When the Length of a Vacation Follows an Expo- nential Distribution}

Let us investigate the queue size and the system time when the length of vacation follows an exponential distribution. In Section 7.1, the PGF for the queue size and the LST for the system time are derived. A numerical example is presented in Section 7.2.

\subsection{Queue Size and System Time}

If the length of vacation follows an exponential distribution with mean $1 / \eta$, the PGF $\Phi(z)$ in (34) for the queue size $X$ at an arbitrary time is free from Bromwich integral and it is reduced to

$$
\begin{aligned}
\Phi(z)= & \frac{\eta(1-\lambda b)[1-\psi(\eta)]\{\eta+\lambda[1-\tau(\eta)]\}}{[1-\psi(\eta)]\{\eta+\lambda[1-\tau(\eta)]\}-b \lambda \eta[1-\tau(\eta)] \psi(\eta)} \\
& \times\left(\Omega(z)+\frac{z[\eta \Omega(z)-1][1-\beta(\lambda-\lambda z)]}{\lambda(1-z)[z-\beta(\lambda-\lambda z)]}\right)
\end{aligned}
$$


where

$$
\Omega(z):=\frac{z[1-\psi(\eta+\lambda-\lambda z)]+(z-1) \psi(\eta+\lambda-\lambda z) \frac{\eta+\lambda(1-z)}{\eta+\lambda[1-\tau(\eta)]}}{[\eta+\lambda(1-z)][z-\psi(\eta+\lambda-\lambda z)]} .
$$

After lengthy elementary manipulation, the mean $\mathrm{E}[X]$ and the variance $\operatorname{Var}[X]$ are given by

$$
\begin{aligned}
\mathrm{E}[X]= & \frac{1}{2 \eta(1-\lambda b)[1-\psi(\eta)](\lambda\{\tau(\eta)+(1+\eta b)[1-\tau(\eta)] \psi(\eta)\}-\eta[1-\psi(\eta)]-\lambda)} \\
& \times \lambda\left[\lambda^{2}\left[2 b(1+b \eta)-b_{2} \eta\right][1-\tau(\eta)][1-\psi(\eta)]^{2}-2 b \eta^{2}\left[1-\tau(\eta) \psi(\eta)^{2}\right](1-\lambda b)\right. \\
& -[1-\psi(\eta)]\left(2 \lambda+\eta\left(2+\lambda \eta b_{2}\right)-2\left[\lambda+\eta\left(2+3 \eta b-\lambda b-3 \lambda \eta b^{2}+\lambda \eta b_{2}\right)\right] \psi(\eta)\right. \\
& \left.-\left\{2 \lambda(1+\eta b)-\left[2 \lambda+\eta\left(2+\lambda \eta b_{2}\right)\right] \psi(\eta)\right\} \tau(\eta)\right) \\
& \left.-2 \lambda \eta^{2} b[1-\tau(\eta)](1-\lambda b) \psi^{\prime}(\eta)\right]
\end{aligned}
$$

and

$$
\begin{aligned}
& \operatorname{Var}[X]=\frac{1}{(\eta+\lambda-(\eta+\lambda+b \eta \lambda) \psi(\eta)+\lambda \tau(\eta)(-1+\psi(\eta)+b \eta \psi(\eta)))^{2}} \\
& \times \frac{1}{12 \eta^{2}(-1+b \lambda)^{2}(-1+\psi(\eta))^{2}}\left(\lambda \left(-3 \lambda\left(\eta \lambda b_{2}(-1+\psi(\eta))(\eta+\lambda-(2 \eta+\lambda) \psi(\eta)\right.\right.\right. \\
& +\tau(\eta)(-\lambda+(\eta+\lambda) \psi(\eta)))+2(-1+b \lambda)((1+b \eta)(\eta+\lambda) \\
& +\psi(\eta)(-((1+b \eta)(3 \eta+2 \lambda))+(\lambda+\eta(2+3 b \eta+b \lambda)) \psi(\eta)) \\
& +b \eta^{2} \lambda \psi^{\prime}(\eta)-\tau(\eta)(\lambda+b \eta \lambda-(\eta+2 \lambda+2 b \eta \lambda) \psi(\eta) \\
& \left.\left.\left.+(1+b \eta)(\eta+\lambda) \psi(\eta)^{2}+b \eta^{2} \lambda \psi^{\prime}(\eta)\right)\right)\right)^{2}+2(\eta+\lambda \\
& -(\eta+\lambda+b \eta \lambda) \psi(\eta)+\lambda \tau(\eta)(-1+\psi(\eta)+b \eta \psi(\eta))) \\
& \times\left(6(1+b \eta)(\eta+\lambda)(\eta+2 \lambda)(-1+b \lambda)^{2}+3 \eta^{2} \lambda^{3} b_{2}^{2}(-1+\psi(\eta))^{2}\right. \\
& \times(\eta+\lambda-(2 \eta+\lambda) \psi(\eta)+\tau(\eta)(-\lambda+(\eta+\lambda) \psi(\eta))) \\
& +3 \eta \lambda(-1+b \lambda) b_{2}(-1+\psi(\eta))\left((\eta+\lambda)(3 \eta+2 \lambda)-(3 \eta+2 \lambda)^{2} \psi(\eta)\right. \\
& +\left(8 \eta^{2}+7 \eta \lambda+2 \lambda^{2}\right) \psi(\eta)^{2}+2 \eta^{2} \lambda \psi^{\prime}(\eta)-\tau(\eta)\left(-\left(\left(\eta^{2}+8 \eta \lambda+4 \lambda^{2}\right) \psi(\eta)\right)\right. \\
& \left.\left.+(\eta+\lambda)(3 \eta+2 \lambda) \psi(\eta)^{2}+\lambda\left(3 \eta+2 \lambda+2 \eta^{2} \psi^{\prime}(\eta)\right)\right)\right) \\
& +2(-1+b \lambda)\left(-\left(\eta^{2} \lambda^{2} b_{3}(-1+\psi(\eta))^{2}(\eta+\lambda-(2 \eta+\lambda) \psi(\eta)\right.\right. \\
& +\tau(\eta)(-\lambda+(\eta+\lambda) \psi(\eta))))+3(-1+b \lambda)\left(\left(\eta^{2}(7+6 b \eta)+13 \eta(1+b \eta) \lambda\right.\right. \\
& \left.+6(1+b \eta) \lambda^{2}\right) \psi(\eta)^{2}-\left(\eta^{2}(4+5 b \eta)+5 \eta(1+b \eta) \lambda+2(1+b \eta) \lambda^{2}\right) \psi(\eta)^{3} \\
& +\eta^{2} \lambda\left((2+3 b \eta) \psi^{\prime}(\eta)-2 b \eta \lambda \psi^{\prime}(\eta)^{2}-b \eta \lambda \psi^{\prime \prime}(\eta)\right) \\
& +\psi(\eta)\left(-((1+b \eta)(\eta+2 \lambda)(4 \eta+3 \lambda))+\eta^{2} \lambda\left(-\left((2+7 b \eta) \psi^{\prime}(\eta)\right)\right.\right. \\
& \left.\left.+b \eta \lambda \psi^{\prime \prime}(\eta)\right)\right)+\tau(\eta)\left(-((1+b \eta) \lambda(\eta+2 \lambda))+\left(b \eta^{3}-7 \eta(1+b \eta) \lambda\right.\right. \\
& \left.-6(1+b \eta) \lambda^{2}\right) \psi(\eta)^{2}+(1+b \eta)(\eta+\lambda)(\eta+2 \lambda) \psi(\eta)^{3} \\
& +\eta^{2} \lambda\left(\psi^{\prime}(\eta)\left(-2-b \eta+2 b \eta \lambda \psi^{\prime}(\eta)\right)+b \eta \lambda \psi^{\prime \prime}(\eta)\right)+\psi(\eta)\left(-\eta^{2}+5 \eta(1+b \eta) \lambda\right.
\end{aligned}
$$




$$
\left.\left.\left.\left.\left.\left.\left.+6(1+b \eta) \lambda^{2}+\eta^{2} \lambda\left((2+5 b \eta) \psi^{\prime}(\eta)-b \eta \lambda \psi^{\prime \prime}(\eta)\right)\right)\right)\right)\right)\right)\right)\right) .
$$

In the same way as deriving (44), we obtain through (43) the LST of the distribution function for the arbitrary customer's system time $T$ as

$$
\begin{aligned}
\alpha(s)= & \frac{\eta(1-\lambda b)[1-\psi(\eta)]\{\eta+\lambda[1-\tau(\eta)]\}}{[1-\psi(\eta)]\{\eta+\lambda[1-\tau(\eta)]\}-b \lambda \eta[1-\tau(\eta)] \psi(\eta)} \\
& \times\left(\omega(s)+\frac{(\lambda-s)[\eta \omega(s)-1][1-\beta(s)]}{s\{\lambda[1-\beta(s)]-s\}}\right),
\end{aligned}
$$

where

$$
\omega(s):=\Omega(1-s / \lambda)=\frac{(\lambda-s)[1-\psi(s+\eta)]-s \psi(s+\eta) \frac{s+\eta}{\eta+\lambda[1-\tau(\eta)]}}{(s+\eta)\{\lambda[1-\psi(s+\eta)]-s\}} .
$$

Thus the mean $\mathrm{E}[T]$ and the variance $\operatorname{Var}[T]$ are given by

$$
\begin{aligned}
\mathrm{E}[T]= & \frac{\mathrm{E}[X]}{\lambda} \\
= & \frac{1}{2 \eta(1-\lambda b)[1-\psi(\eta)](\lambda\{\tau(\eta)+(1+\eta b)[1-\tau(\eta)] \psi(\eta)\}-\eta[1-\psi(\eta)]-\lambda)} \\
& \times\left[\lambda^{2}\left[2 b(1+b \eta)-b_{2} \eta\right][1-\tau(\eta)][1-\psi(\eta)]^{2}-2 b \eta^{2}\left[1-\tau(\eta) \psi(\eta)^{2}\right](1-\lambda b)\right. \\
& -[1-\psi(\eta)]\left(2 \lambda+\eta\left(2+\lambda \eta b_{2}\right)-2\left[\lambda+\eta\left(2+3 \eta b-\lambda b-3 \lambda \eta b^{2}+\lambda \eta b_{2}\right)\right] \psi(\eta)\right. \\
& \left.-\left\{2 \lambda(1+\eta b)-\left[2 \lambda+\eta\left(2+\lambda \eta b_{2}\right)\right] \psi(\eta)\right\} \tau(\eta)\right) \\
& \left.-2 \lambda \eta^{2} b[1-\tau(\eta)](1-\lambda b) \psi^{\prime}(\eta)\right]
\end{aligned}
$$

and

$$
\begin{aligned}
\operatorname{Var}[T]= & \frac{1}{(\eta+\lambda-(\eta+\lambda+b \eta \lambda) \psi(\eta)+\lambda \tau(\eta)(-1+\psi(\eta)+b \eta \psi(\eta)))^{2}} \\
& \times \frac{1}{12 \eta^{2} \lambda(-1+b \lambda)^{2}(-1+\psi(\eta))^{2}}\left(3 \eta^{2} \lambda^{3} b_{2}^{2}(-1+\psi(\eta))^{2}(\eta+\lambda-(2 \eta+\lambda) \psi(\eta)\right. \\
& +\tau(\eta)(-\lambda+(\eta+\lambda) \psi(\eta)))(\eta+\lambda-(\lambda+2 b \eta \lambda) \psi(\eta) \\
& +\tau(\eta)(-\lambda+(-\eta+\lambda+2 b \eta \lambda) \psi(\eta)))-12 \eta^{2} \lambda(-1+b \lambda)^{2} b_{2} \\
& \times(-1+\psi(\eta))^{2}\left((\eta+\lambda)(3 \eta+\lambda) \psi(\eta)-\eta(3 \eta+2 \lambda) \psi(\eta)^{2}\right. \\
& +\lambda^{2} \tau(\eta)^{2}\left(-1+\psi(\eta)-\eta \psi^{\prime}(\eta)\right)-(\eta+\lambda)\left(\eta+\lambda+\eta \lambda \psi^{\prime}(\eta)\right) \\
& +\tau(\eta)\left(2 \lambda(\eta+\lambda)-2 \lambda(2 \eta+\lambda) \psi(\eta)+\eta(\eta+2 \lambda) \psi(\eta)^{2}\right. \\
& \left.\left.+\eta \lambda(\eta+2 \lambda) \psi^{\prime}(\eta)\right)\right)-4(-1+b \lambda)\left(\eta^{2} \lambda^{2} b_{3}(-1+\psi(\eta))^{2}(\eta+\lambda\right. \\
& -(\eta+\lambda+b \eta \lambda) \psi(\eta)+\lambda \tau(\eta)(-1+\psi(\eta)+b \eta \psi(\eta)))(\eta+\lambda \\
& -(2 \eta+\lambda) \psi(\eta)+\tau(\eta)(-\lambda+(\eta+\lambda) \psi(\eta)))+3(-1+b \lambda)((-1+b \eta) \\
& \times(1+b \eta) \lambda(\eta+\lambda)^{2}+2\left(\eta^{3}(2+b \eta)-3 \eta^{2}(1+b \eta)(-1+3 b \eta) \lambda\right.
\end{aligned}
$$




$$
\begin{aligned}
& \left.-4 \eta\left(-1+b^{2} \eta^{2}\right) \lambda^{2}+(1+b \eta)(2+b \eta) \lambda^{3}\right) \psi(\eta)^{3}+\left(-2 \eta^{3}(1+b \eta)\right. \\
& \left.+\eta^{2}(-2+b \eta(4+7 b \eta)) \lambda+2 \eta\left(-1+b^{2} \eta^{2}\right) \lambda^{2}-(1+b \eta)^{2} \lambda^{3}\right) \psi(\eta)^{4} \\
& +\eta^{2} \lambda\left(2(1+b \eta)(\eta+\lambda)(-1+b \lambda) \psi^{\prime}(\eta)+b \eta \lambda(2 \eta+2 \lambda+b \eta \lambda) \psi^{\prime}(\eta)^{2}\right. \\
& \left.+b \eta \lambda(\eta+\lambda) \psi^{\prime \prime}(\eta)\right)+\tau(\eta)\left(2 \left(-\left((-1+b \eta)(1+b \eta) \lambda^{2}(\eta+\lambda)\right)\right.\right. \\
& +\psi(\eta)\left(\eta^{3}+\eta^{2} \lambda+4 \eta\left(-1+b^{2} \eta^{2}\right) \lambda^{2}+2(-2+b \eta)(1+b \eta) \lambda^{3}\right. \\
& +\psi(\eta)\left(-2 \eta^{2} \lambda+6 \lambda^{3}-b \eta^{4}(1+b \lambda)+6 \eta \lambda^{2}(1+b \lambda)-2 \eta^{3}(1+b \lambda(2+3 b \lambda))\right. \\
& +\psi(\eta)\left(\eta^{3}(1+b \eta)+\eta^{2}(1+2 b \eta(3+2 b \eta)) \lambda+4 \eta\left(-1+b^{2} \eta^{2}\right) \lambda^{2}\right. \\
& \left.\left.\left.-2(1+b \eta)(2+b \eta) \lambda^{3}-(1+b \eta) \lambda(\eta+\lambda)\left(2 b \eta^{2}-(1+b \eta) \lambda\right) \psi(\eta)\right)\right)\right) \\
& +\eta^{2} \lambda\left(\eta+2 \lambda-b^{2} \eta^{2} \lambda-2 b(1+b \eta) \lambda^{2}+\psi(\eta)(-2 \eta(1+b \eta)+2(-2+b \eta(-2+b \eta)) \lambda\right. \\
& \left.\left.+4 b(1+b \eta) \lambda^{2}+\left(\eta(1+2 b \eta)+(2+b \eta(4+b \eta)) \lambda-2 b(1+b \eta) \lambda^{2}\right) \psi(\eta)\right)\right) \psi^{\prime}(\eta) \\
& \left.+b \eta^{3} \lambda^{2}(-\eta-2 \lambda-b \eta \lambda+(\eta+2 \lambda+2 b \eta \lambda) \psi(\eta)) \psi^{\prime}(\eta)^{2}\right) \\
& \left.-b \eta^{3} \lambda^{2}(-1+\psi(\eta))(-\eta-2 \lambda+(\eta+2 \lambda+2 b \eta \lambda) \psi(\eta)) \psi^{\prime \prime}(\eta)\right) \\
& +\psi(\eta)^{2}\left(-2 \eta^{3}+\eta^{2}(-7+5 b \eta(2+3 b \eta)) \lambda+12 \eta\left(-1+b^{2} \eta^{2}\right) \lambda^{2}-6(1+b \eta) \lambda^{3}\right. \\
& \left.+\eta^{2} \lambda\left(-2\left(\eta(1+3 b \eta)+\lambda+2 b \eta \lambda-b(1+b \eta) \lambda^{2}\right) \psi^{\prime}(\eta)+b \eta \lambda(\eta+\lambda+b \eta \lambda) \psi^{\prime \prime}(\eta)\right)\right) \\
& +\lambda \psi(\eta)(-2(1+b \eta)(\eta+\lambda)(-2 \lambda+\eta(-2+b(3 \eta+\lambda))) \\
& +\eta^{2}\left(-4(\eta+\lambda)(-1+b(-2 \eta+\lambda+b \eta \lambda)) \psi^{\prime}(\eta)-2 b \eta \lambda(\eta+\lambda+b \eta \lambda) \psi^{\prime}(\eta)^{2}\right. \\
& \left.\left.-b \eta \lambda(2 \eta+2 \lambda+b \eta \lambda) \psi^{\prime \prime}(\eta)\right)\right)+\lambda \tau(\eta)^{2}\left(-2(1+b \eta)(2+b \eta)(\eta-\lambda)(\eta+\lambda) \psi(\eta)^{3}\right. \\
& +(1+b \eta)^{2}(\eta-\lambda)(\eta+\lambda) \psi(\eta)^{4}+\psi(\eta)^{2}\left(\eta^{2}(5+4 b \eta)-6(1+b \eta) \lambda^{2}\right. \\
& \left.+\eta^{2}(1+b \eta) \lambda\left(-2(1+b \eta-b \lambda) \psi^{\prime}(\eta)+b \eta \lambda \psi^{\prime \prime}(\eta)\right)\right)+\lambda\left(\left(-1+b^{2} \eta^{2}\right) \lambda\right. \\
& \left.+\eta^{2}\left(\psi^{\prime}(\eta)\left(-2+2 b(1+b \eta) \lambda+b \eta(2+b \eta) \lambda \psi^{\prime}(\eta)\right)+b \eta \lambda \psi^{\prime \prime}(\eta)\right)\right) \\
& +\psi(\eta)\left(4 \lambda^{2}-2 \eta\left(\eta+b(-1+b \eta) \lambda^{2}\right)+\eta^{2} \lambda\left(-2(1+b \eta) \psi^{\prime}(\eta)\left(-2+2 b \lambda+b \eta \lambda \psi^{\prime}(\eta)\right)\right.\right. \\
& \left.\left.\left.\left.\left.-b \eta(2+b \eta) \lambda \psi^{\prime \prime}(\eta)\right)\right)\right)\right)\right) .
\end{aligned}
$$

\section{Remark}

As shown in Appendices B and C, two special cases can be derived from (47). (i) If the server is always on a working vacation, i.e., $\eta \rightarrow 0$, (47) is reduced to

$$
\Phi(z)=\frac{(1-\lambda h)(1-z) \psi(\lambda-\lambda z)}{\psi(\lambda-\lambda z)-z},
$$

where $h$ is the mean of the service time in a working vacation. (ii) If the server never takes a vacation, i.e., $\eta \rightarrow \infty$, (47) becomes

$$
\Phi(z)=\frac{(1-\lambda b)(1-z) \beta(\lambda-\lambda z)}{\beta(\lambda-\lambda z)-z} .
$$

Each of (55) and (56) is referred to as the Pollaczek-Khinchin transform equation for an $\mathrm{M} / \mathrm{G} / 1$ queue $[10, \mathrm{p} .6$, eq. (1.18)]. 


\subsection{Numerical Example}

In this section we illustrate the results obtained in Section 7.1 numerically. In our numerical example, we assume that service time in a working vacation follows an Erlang-2 distribution with mean 1 for which the LST is given by

$$
\psi(s)=\left(\frac{2}{s+2}\right)^{2} .
$$

The service time during a service period is assumed to follow an Erlang-3 distribution with mean 0.2 for which the LST is given by

$$
\beta(s)=\left(\frac{15}{s+15}\right)^{3} .
$$

Combining (3) and (57) yields the equation

$$
\tau(\eta)=\left(\frac{2}{\eta+\lambda[1-\tau(\eta)]+2}\right)^{2},
$$

which we solve for $\tau(\eta)$ numerically.

We plot the performance values by changing the arrival rate $\lambda$. In order to investigate the influence of the mean for the length of a vacation $v=1 / \eta$, we show the results for several values of $v$. Figure 2 and Figure 3 show the mean and variance, respectively, for the queue size. Figure 4 and Figure 5 show the mean and variance, respectively, for the system time. For comparison, the performance values for two $M / E_{r} / 1$ queues whose LSTs of the service time distributions are given by (57) and (58), respectively, are also plotted. It is observed that at large and small values of $v$ our system behaves like an $\mathrm{M} / \mathrm{E}_{\mathrm{r}} / 1$ queue in accordance to the limits given in (55) and (56).

\section{When the Service Time in a Vacation Follows an Exponential Distribution}

In this section we consider the special situation in which the service time in a vacation follows an exponential distribution. In Section 8.1 the queue size and the system time are derived. Numerical examples are given in Section 8.2.

\subsection{Queue Size and System time}

If the service time in a vacation follows an exponential distribution with rate $\nu$, we have

$$
\psi(s)=\frac{\nu}{s+\nu}
$$

From (3) and (60), the LST $\tau(s)$ included in $\Phi(z)$ in (34) satisfies the equation

$$
\tau(s)=\frac{\nu}{s+\lambda-\lambda \tau(s)+\nu}
$$




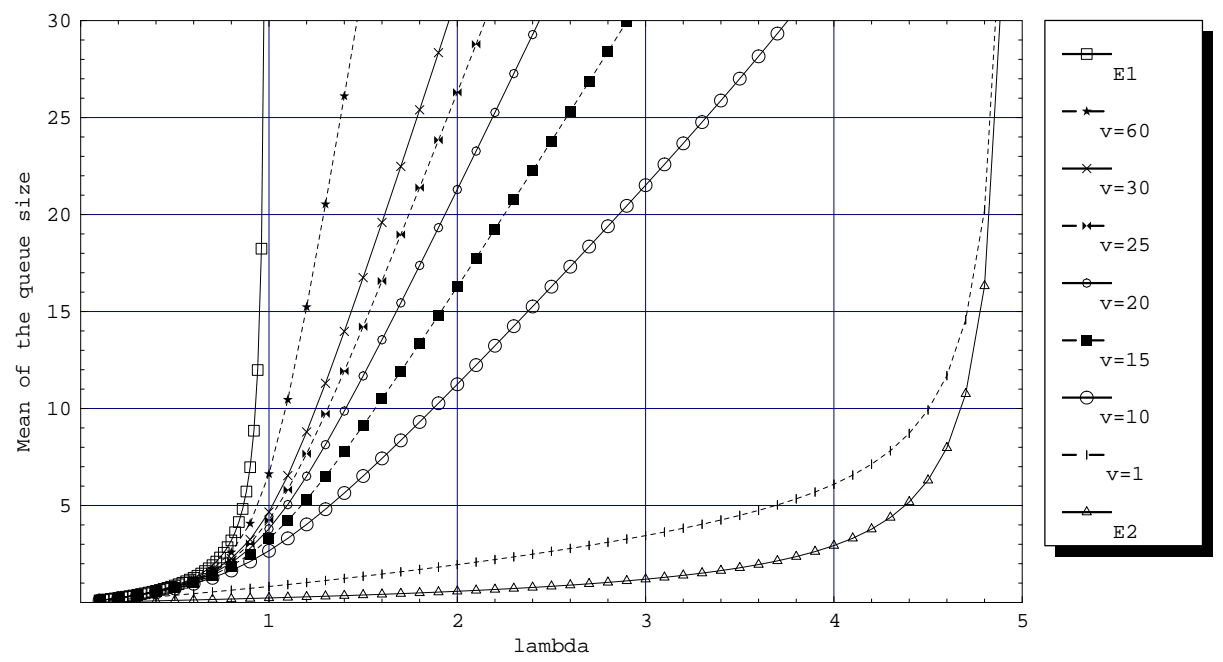

Figure 2: Mean of the queue size when the service times in a working vacation and in a service period follow Erlang distribution with mean 1, order 2 and mean 0.5, order 3 , respectively, and the length of a vacation is exponentially distributed with mean $v$. $\mathrm{E} 1$ and $\mathrm{E} 2$ denote two $\mathrm{M} / \mathrm{E}_{\mathrm{r}} / 1$ queues and their service time distributions have mean 1, order 2 and mean 0.5 , order 3 , respectively.

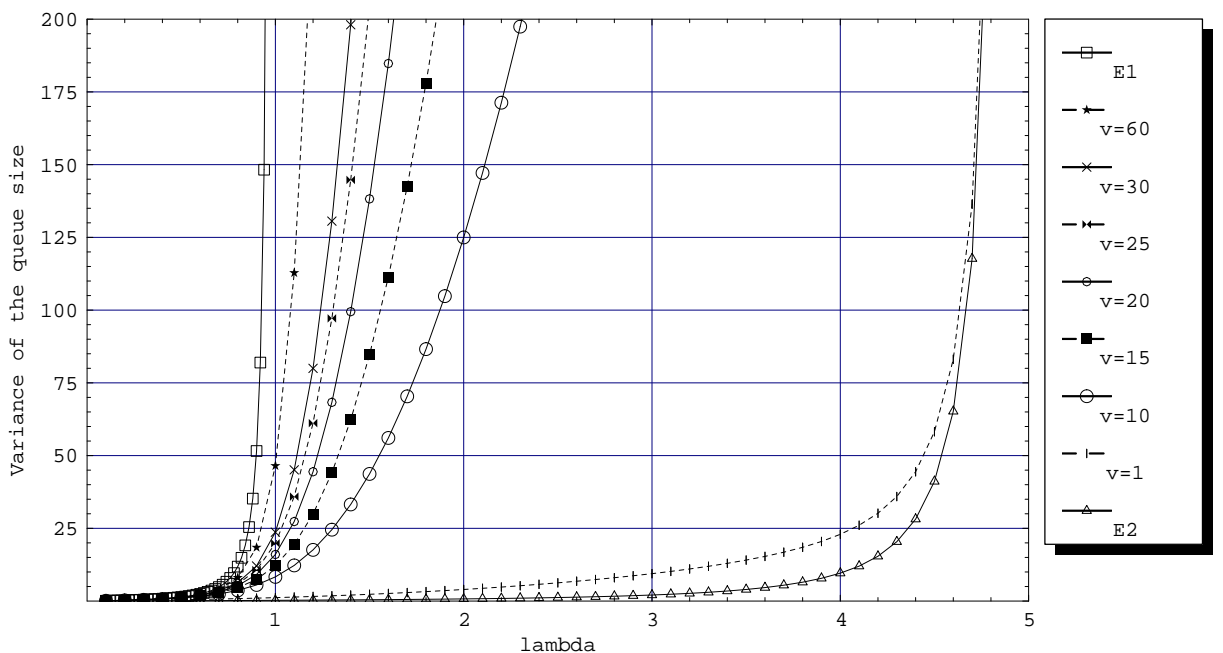

Figure 3: Variance of the queue size when the service times in a working vacation and in a service period follow Erlang distribution with mean 1, order 2 and mean 0.5 , order 3 , respectively, and the length of a vacation is exponentially distributed with mean $v$. $\mathrm{E} 1$ and $\mathrm{E} 2$ denote two $\mathrm{M} / \mathrm{E}_{\mathrm{r}} / 1$ queues and their service time distributions have mean 1, order 2 and mean 0.5 , order 3 , respectively. 


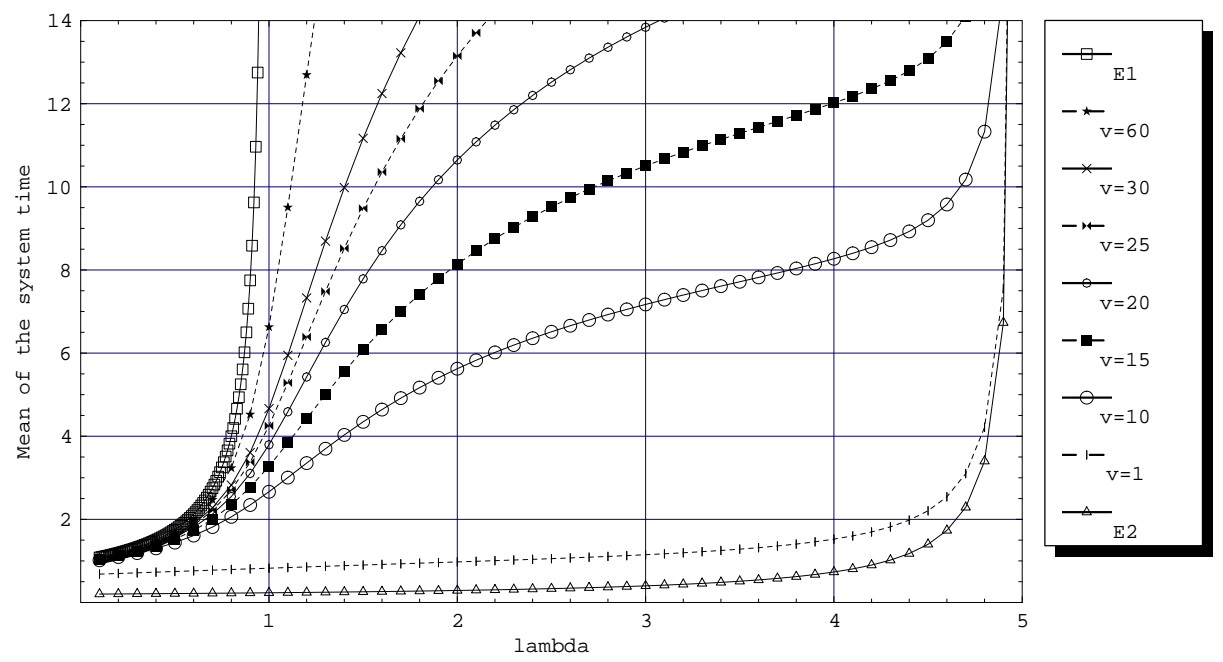

Figure 4: Mean of the system time when the service times in a working vacation and in a service period follow Erlang distribution with mean 1, order 2 and mean 0.5, order 3, respectively, and the length of a vacation is exponentially distributed with mean $v$. E1 and $\mathrm{E} 2$ denote $\mathrm{M} / \mathrm{E}_{\mathrm{r}} / 1$ queues and their service time distributions have mean 1 , order 2 and mean 0.5 , order 3 , respectively.

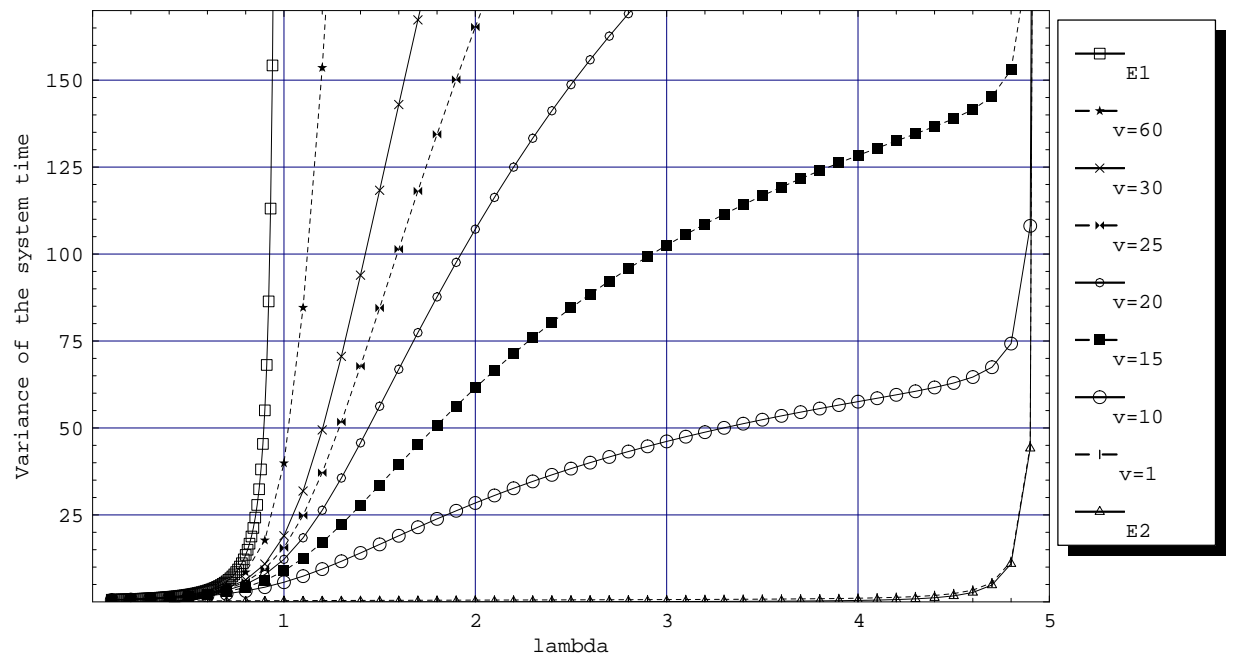

Figure 5: Variance of the system time when the service times in a working vacation and in a service period follow Erlang distribution with mean 1, order 2 and mean 0.5 , order 3 , respectively, and the length of a vacation is exponentially distributed with mean $v$. $\mathrm{E} 1$ and $\mathrm{E} 2$ denote two $\mathrm{M} / \mathrm{E}_{\mathrm{r}} / 1$ queues and their service time distributions have mean 1, order 2 and mean 0.5 , order 3 , respectively. 
which may be written as

$$
\frac{1}{s+\lambda-\lambda \tau(s)}=\frac{\tau(s)}{\nu(1-\tau(s))}
$$

Solving (61) yields $\tau(s)=\tau_{1}(s)$ and $\tau_{2}(s)$, where

$$
\begin{aligned}
\tau_{1}(s) & :=\frac{s+\lambda+\nu+\sqrt{(s+\lambda+\nu)^{2}-4 \lambda \nu}}{2 \lambda} \\
\tau_{2}(s) & :=\frac{s+\lambda+\nu-\sqrt{(s+\lambda+\nu)^{2}-4 \lambda \nu}}{2 \lambda} .
\end{aligned}
$$

We claim that $\tau_{2}(s)$ is the LST of the distribution function for the length of a busy period in a working vacation, since $\tau_{2}(s)$ is the only solution to the equation in $(61)$ such that $|\tau(s)| \leq 1$. Now, by substituting (60) into (2) and using relation (62) we have

$$
\gamma_{i}(z, s)=\frac{z^{i+1}+(z-1) \nu P_{i, 0}^{*}(s)}{z s-(1-z)(\nu-\lambda z)} ; \quad i=0,1,2, \ldots
$$

where

$$
P_{i, 0}^{*}(s)=\frac{\left[\tau_{2}(s)\right]^{i+1}}{\nu\left[1-\tau_{2}(s)\right]}
$$

From (4) the LT of the expected number of customers served in the time interval $(0, t]$ with initial queue size $X(0)=i(\geq 0)$ is given by

$$
N_{i}^{*}(s)=\frac{\nu}{s^{2}}-\frac{\left[\tau_{2}(s)\right]^{i+1}}{s\left[1-\tau_{2}(s)\right]} ; \quad i=0,1,2, \ldots
$$

Thus the expected number of customers served in a vacation defined in (15) is

$$
\begin{aligned}
N & =\frac{1}{2 \pi \mathbf{i}} \int_{B r} N_{0}^{*}(s) \phi(-s) d s=\int_{0}^{\infty} d V(t) \frac{1}{2 \pi \mathbf{i}} \int_{B r}\left(\frac{\nu}{s^{2}}-\frac{\tau_{2}(s)}{s\left[1-\tau_{2}(s)\right]}\right) e^{s t} d s \\
& =\nu \int_{0}^{\infty}\left(t-\int_{0}^{t} P_{0,0}(x) d x\right) d V(t)=\nu\left(v-E_{1}\right),
\end{aligned}
$$

where we have used the relation (65), and we have defined

$$
E_{n}:=\int_{0}^{\infty}[\underbrace{\int_{0}^{t} \int_{0}^{t} \cdots \int_{0}^{t}}_{n} P_{0,0}(x)(d x)^{n}] d V(t) ; \quad n=1,2, \ldots
$$

Equivalently, $E_{n}$ can be rewritten as

$$
E_{n}=\frac{1}{2 \pi \mathbf{i}} \int_{B r} \frac{\tau_{2}(s)}{s^{n} \nu\left[1-\tau_{2}(s)\right]} \phi(-s) d s
$$

Note that $E_{1}$ is the sum of the mean lengths of those periods during which the queue is empty in a vacation time. 
Substituting (64) and (67) into (34), we obtain the PGF for the queue size at an arbitrary time

$$
\Phi(z)=\frac{1-\lambda b}{v-\nu b\left(v-E_{1}\right)}\left[v \hat{\Psi}(z)+\frac{z[\Psi(z)-1][1-\beta(\lambda-\lambda z)]}{\lambda(1-z)[z-\beta(\lambda-\lambda z)]}\right]
$$

where

$$
\Psi(z)=\frac{1}{2 \pi \mathbf{i}} \int_{B r}\left[\frac{z+(z-1) \frac{\tau_{2}(s)}{1-\tau_{2}(s)}}{z s-(1-z)(\nu-\lambda z)}\right] \phi(-s) d s
$$

and

$$
\hat{\Psi}(z)=\frac{1}{2 \pi \mathbf{i}} \int_{B r}\left[\frac{z+(z-1) \frac{\tau_{2}(s)}{1-\tau_{2}(s)}}{z s-(1-z)(\nu-\lambda z)}\right] \hat{\phi}(-s) d s .
$$

Differentiating (70) and using L'Hospital's rule four times at $z=1$ yields

$$
\begin{aligned}
\mathrm{E}[X]= & \frac{1}{2(1-\lambda b)\left[v-\nu b\left(v-E_{1}\right)\right]}\left\{2 \lambda v b(1-\lambda b)+\lambda b_{2}\left[\lambda v-\nu\left(v-E_{1}\right)\right]\right. \\
& \left.+(1-\lambda b)(1-\nu b)\left[v_{2}(\lambda-\nu)+2 \nu E_{2}\right]\right\}
\end{aligned}
$$

where we have used

$$
\begin{aligned}
\Psi^{\prime}(1)= & \frac{1}{2 \pi \mathbf{i}} \int_{B r}\left[\frac{\lambda-\nu}{s^{2}}+\nu\left(\frac{\tau_{2}(s)}{s \nu\left[1-\tau_{2}(s)\right]}\right)\right] \phi(-s) d s \\
= & \lambda v-N=\lambda v-\nu\left(v-E_{1}\right), \\
\Psi^{\prime \prime}(1)= & \frac{1}{2 \pi \mathbf{i}} \int_{B r}\left[2 \frac{(\lambda-\nu)^{2}}{s^{3}}+\frac{2 \nu}{s^{2}}-2 \nu\left(\frac{\tau_{2}(s)}{s \nu\left[1-\tau_{2}(s)\right]}\right)\right. \\
& \left.+2 \nu(\lambda-\nu)\left(\frac{\tau_{2}(s)}{s^{2} \nu\left[1-\tau_{2}(s)\right]}\right)\right] \phi(-s) d s \\
= & (\lambda-\nu)^{2} v_{2}+2 \nu\left(v-E_{1}\right)+2 \nu(\lambda-\nu) E_{2},
\end{aligned}
$$

and

$$
\begin{aligned}
\hat{\Psi}^{\prime}(1) & =\frac{1}{2 \pi \mathbf{i}} \int_{B r}\left[\frac{\lambda-\nu}{s^{2}}+\nu\left(\frac{\tau_{2}(s)}{s \nu\left[1-\tau_{2}(s)\right]}\right)\right] \hat{\phi}(-s) d s \\
& =\frac{1}{v} \int_{0}^{\infty}[1-V(t)] d t \frac{1}{2 \pi \mathbf{i}} \int_{B r}\left[\frac{\lambda-\nu}{s^{2}}+\nu\left(\frac{\tau_{2}(s)}{s \nu\left[1-\tau_{2}(s)\right]}\right)\right] e^{s t} d s \\
& =\frac{1}{v} \int_{0}^{\infty}[1-V(t)]\left[(\lambda-\nu) t+\nu \int_{0}^{t} P_{0,0}(x) d x\right] d t \\
& =\frac{(\lambda-\nu) v_{2}}{2 v}+\frac{\nu E_{2}}{v} .
\end{aligned}
$$

Differentiating (70) twice and using L'Hospital's rule six times at $z=1$, we obtain

$$
\operatorname{Var}[X]=\frac{-1}{12(-1+b \lambda)^{2}\left(v+b\left(-v+E_{1}\right) \nu\right)^{2}}\left(3 \left(-2 b v \lambda(-1+b \lambda)+\lambda b_{2}(v \lambda\right.\right.
$$




$$
\begin{aligned}
& \left.\left.+\left(-v+E_{1}\right) \nu\right)+(-1+b \lambda)(-1+b \nu)\left(v_{2}(\lambda-\nu)+2 E_{2} \nu\right)\right)^{2} \\
& +2\left(-v+b\left(v-E_{1}\right) \nu\right)\left(3 \lambda^{3} b_{2}^{2}\left(v \lambda+\left(-v+E_{1}\right) \nu\right)-3 \lambda(-1+b \lambda) b_{2}(3 v \lambda\right. \\
& \left.+v_{2}(\lambda-\nu)^{2}+\left(-v+E_{1}+2 E_{2}(\lambda-\nu)\right) \nu\right)+(-1+b \lambda)\left(-2 \lambda^{2} b_{3}(v \lambda\right. \\
& \left.+\left(-v+E_{1}\right) \nu\right)+(-1+b \lambda)\left(-2 v_{3}(\lambda-\nu)^{2}(-1+b \nu)+3 v_{2}(\lambda(1+2 b \lambda)\right. \\
& -\nu(-1+3 b \lambda+b \nu))+6\left(b v \lambda+\nu\left(-2 E_{3}(\lambda-\nu)(-1+b \nu)\right.\right. \\
& \left.\left.\left.\left.\left.+E_{2}(-1+2 b \lambda+b \nu)\right)\right)\right)\right)\right)
\end{aligned}
$$

where we have used

$$
\begin{aligned}
\Psi^{(3)}(1)= & \frac{1}{2 \pi \mathbf{i}} \int_{B r}\left[6 \nu\left(\frac{\tau_{2}(s)}{s \nu\left[1-\tau_{2}(s)\right]}\right)+\frac{6(\lambda-\nu)^{3}}{s^{4}}+12 \frac{\nu(\lambda-\nu)}{s^{3}}\right. \\
& +6 \nu(\lambda-\nu)^{2}\left(\frac{\tau_{2}(s)}{s^{3} \nu\left[1-\tau_{2}(s)\right]}\right)-\frac{6 \nu}{s^{2}} \\
& \left.-6 \nu(\lambda-2 \nu)\left(\frac{\tau_{2}(s)}{s^{2} \nu\left[1-\tau_{2}(s)\right]}\right)\right] \phi(-s) d s \\
= & 6 \nu E_{1}+(\lambda-\nu)^{3} v_{3}+6 \nu(\lambda-\nu) v_{2} \\
& +6 \nu(\lambda-\nu)^{2} E_{3}-6 \nu v-6 \nu(\lambda-2 \nu) E_{2} \\
= & -6 \nu\left(v-E_{1}\right)+(\lambda-\nu)^{2}\left[v_{3}(\lambda-\nu)+6 \nu E_{3}\right] \\
& +6 \nu(\lambda-\nu)\left(v_{2}-E_{2}\right)+6 \nu^{2} E_{2}
\end{aligned}
$$

and

$$
\begin{aligned}
\hat{\Psi}^{\prime \prime}(1)= & \frac{1}{2 \pi \mathbf{i}} \int_{B r}\left[2 \frac{(\lambda-\nu)^{2}}{s^{3}}+\frac{2 \nu}{s^{2}}-2 \nu\left(\frac{\tau_{2}(s)}{s \nu\left[1-\tau_{2}(s)\right]}\right)\right. \\
& \left.+2 \nu(\lambda-\nu)\left(\frac{\tau_{2}(s)}{s^{2} \nu\left[1-\tau_{2}(s)\right]}\right)\right] \hat{\phi}(-s) d s \\
= & \frac{1}{v}\left[\frac{(\lambda-\nu)^{2} v_{3}}{3}+\nu\left(v_{2}-2 E_{2}\right)+2 \nu(\lambda-\nu) E_{3}\right] .
\end{aligned}
$$

In the same way as deriving (44), we obtain the LST of the distribution function for the arbitrary customer's system time $T$ as

$$
\alpha(s)=\frac{1-\lambda b}{v-\nu b\left(v-E_{1}\right)}\left(v \hat{\Psi}(1-s / \lambda)+\frac{(\lambda-s)[\Psi(1-s / \lambda)-1][1-\beta(s)]}{s\{\lambda[1-\beta(s)]-s\}}\right) .
$$

Then $\mathrm{E}[T]$ and $\operatorname{Var}[T]$ are given by

$$
\begin{aligned}
\mathrm{E}[T]=\frac{\mathrm{E}[X]}{\lambda}= & \frac{1}{2 \lambda(1-\lambda b)\left[v-\nu b\left(v-E_{1}\right)\right]}\left\{2 \lambda v b(1-\lambda b)+\lambda b_{2}\left[\lambda v-\nu\left(v-E_{1}\right)\right]\right. \\
& \left.+(1-\lambda b)(1-\nu b)\left[v_{2}(\lambda-\nu)+2 \nu E_{2}\right]\right\}
\end{aligned}
$$

and

$$
\operatorname{Var}[T]=\frac{1}{12 \lambda^{2}(-1+b \lambda)^{2}\left(v+b\left(-v+E_{1}\right) \nu\right)^{2}}(-3(-2 b v \lambda(-1+b \lambda)+
$$




$$
\begin{aligned}
& \left.\lambda b_{2}\left(v \lambda+\left(-v+E_{1}\right) \nu\right)+(-1+b \lambda)(-1+b \nu)\left(v_{2}(\lambda-\nu)+2 E_{2} \nu\right)\right)^{2} \\
& +2\left(-v+b\left(v-E_{1}\right) \nu\right)\left(-3 \lambda^{3} b_{2}^{2}\left(v \lambda+\left(-v+E_{1}\right) \nu\right)+3 \lambda(-1+b \lambda) b_{2}(2 v \lambda\right. \\
& \left.+(\lambda-\nu)\left(v_{2}(\lambda-\nu)+2 E_{2} \nu\right)\right)+2(-1+b \lambda)\left(\lambda^{2} b_{3}\left(v \lambda+\left(-v+E_{1}\right) \nu\right)\right. \\
& +(-1+b \lambda)\left(v_{3}(\lambda-\nu)^{2}(-1+b \nu)-6 \nu\left(-\left(E_{3}(\lambda-\nu)(-1+b \nu)\right)\right.\right. \\
& \left.\left.\left.\left.+E_{2}(-1+b \lambda+b \nu)\right)-3 v_{2}\left(b \lambda^{2}-\nu(-1+b \lambda+b \nu)\right)\right)\right)\right)
\end{aligned}
$$

\section{Remark}

It is shown in Appendix D that if the length of a vacation follows an exponential distribution with mean $1 / \eta$ and the service times in a vacation and that in a service period are exponentially distributed with rate $\nu$ and $\mu$, respectively, $\Phi(z)$ in $(70)$ is reduced to

$$
\Phi(z)=\left(\frac{1-\frac{\lambda}{\mu}}{1-\frac{\lambda}{\mu} z}\right)\left(\frac{1-\tau_{1}(\eta)^{-1}}{1-\tau_{1}(\eta)^{-1} z}\right)\left(\frac{1-\frac{\nu}{\tau_{1}(\eta) \mu}}{1-\frac{\nu}{\tau_{1}(\eta) \mu} z}\right)^{-1},
$$

where $\tau_{1}(\eta)$ is defined in (63). The product form in (83) is the PGF for the queue size at an arbitrary time in an $\mathrm{M} / \mathrm{M} / 1 / \mathrm{WV}$ queue derived and interpreted by Servi and Finn $[8]$.

\subsection{Numerical Examples}

In this section we illustrate the results obtained in Section 8.1 numerically by means of two examples: one is a case in which the length of a vacation is a constant and the other is a case in which the length of a vacation follows an Erlang distribution. For both cases we assume that the service times in a service period and in a vacation are exponentially distributed with rate $\mu=1.5$ and $\mu=3.5$, respectively.

Example 1: Let the length of a vacation be a constant $v$, which means that $\psi(s)=e^{-v s}$. Then we have

$$
E_{n}=\frac{1}{2 \pi \mathbf{i}} \int_{B r} \frac{\tau_{2}(s)}{s^{n} \nu\left[1-\tau_{2}(s)\right]} e^{v s} d s
$$

We may use the algorithm in [1, p.257] to calculate this inverse Laplace transform numerically.

Figure 6 and Figure 7 show the mean and variance, respectively, for the queue size. Figure 8 and Figure 9 show the mean and variance, respectively, for the system time. For comparison, the means and variances of the queue size and the system time in an $\mathrm{M} / \mathrm{M} / 1$ queue are also plotted in the corresponding figures. In both figures, we can observe the influence of the length of a vacation. Our queue in this example behaves like an $\mathrm{M} / \mathrm{M} / 1$ queue as the length of vacation becomes very small or very large. This result agrees with our intuition as well as the limits in (55) and (56).

Example 2: We assume that the length of a vacation follows an Erlang distribution with probability density function

$$
d V(t)=\frac{4}{v^{2}} t e^{\frac{-2 t}{v}} d t
$$




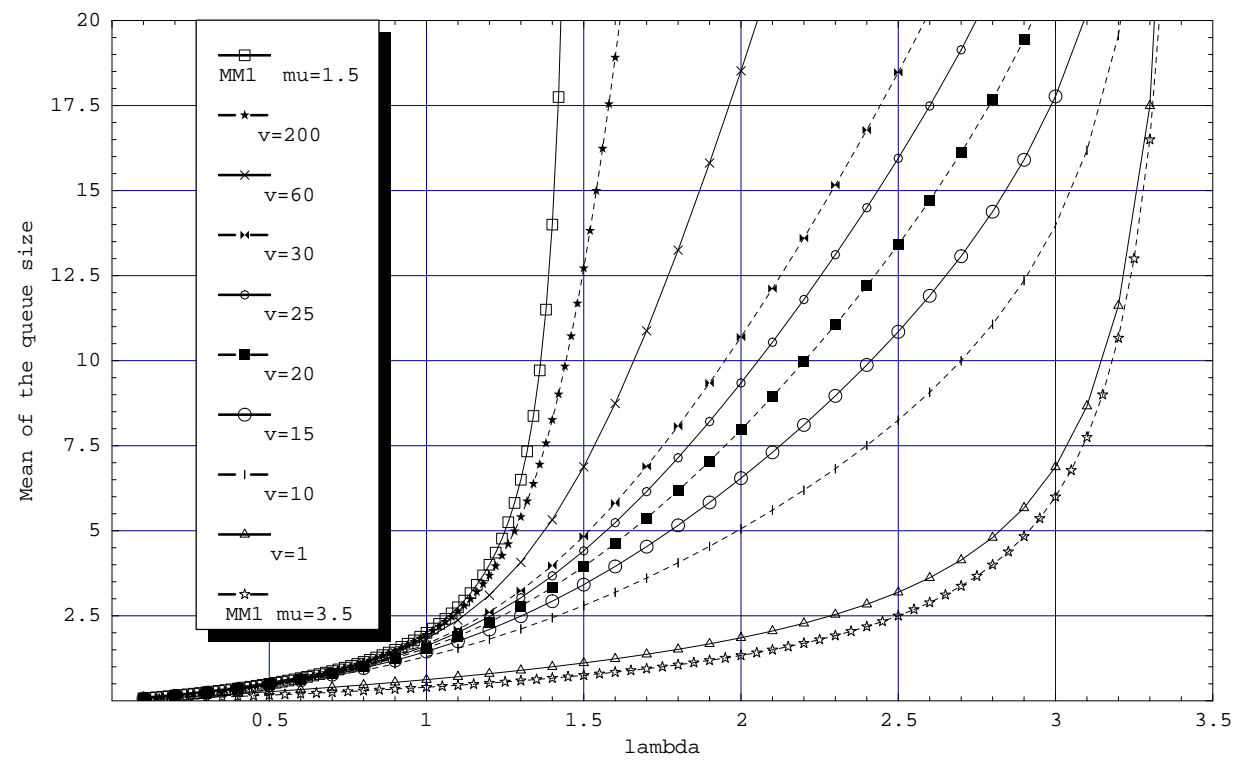

Figure 6: Mean of the queue size when the service times in a working vacation and in a service period are exponentially distributed with $\nu=1.5, \mu=3.5$, respectively, and the length of a vacation is a constant $v$. MM1 means an $\mathrm{M} / \mathrm{M} / 1$ queue.

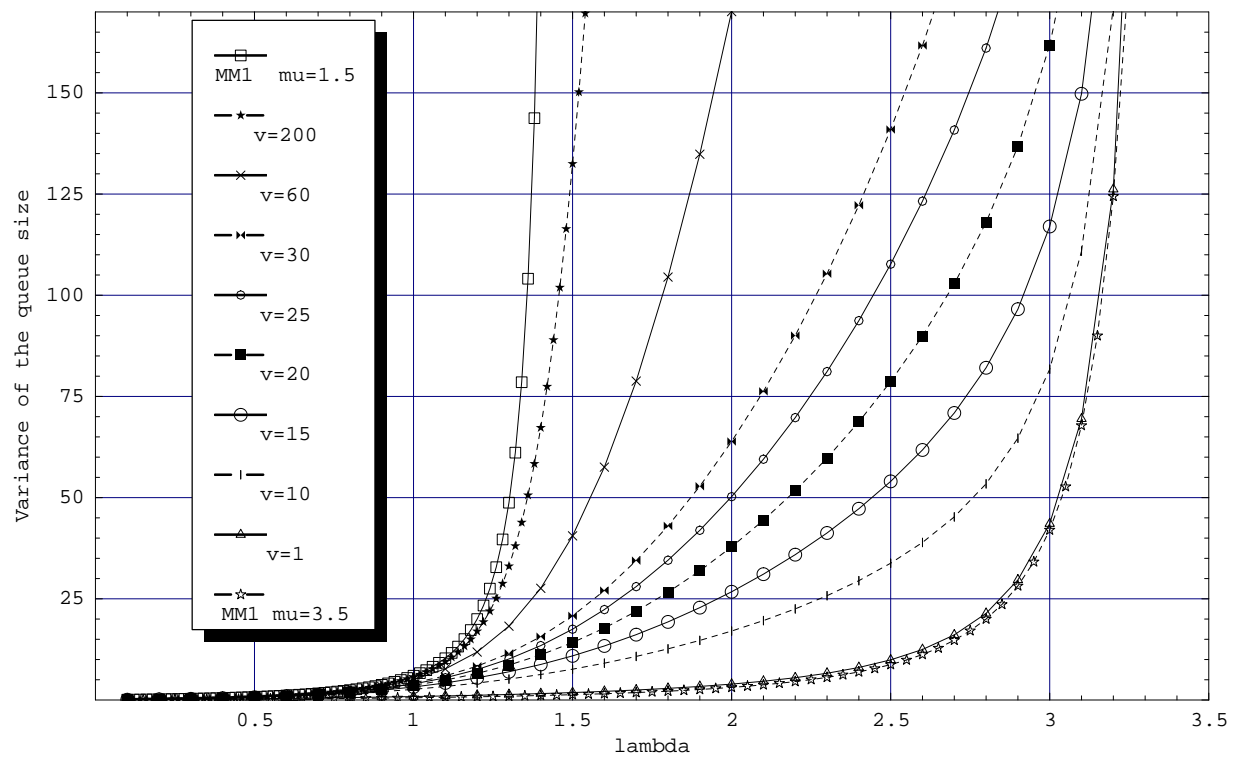

Figure 7: Variance of the queue size when the service times in a working vacation and in a service period are exponentially distributed with $\nu=1.5, \mu=3.5$, respectively, and the length of a vacation is a constant $v$. MM1 means an M/M/1 queue. 


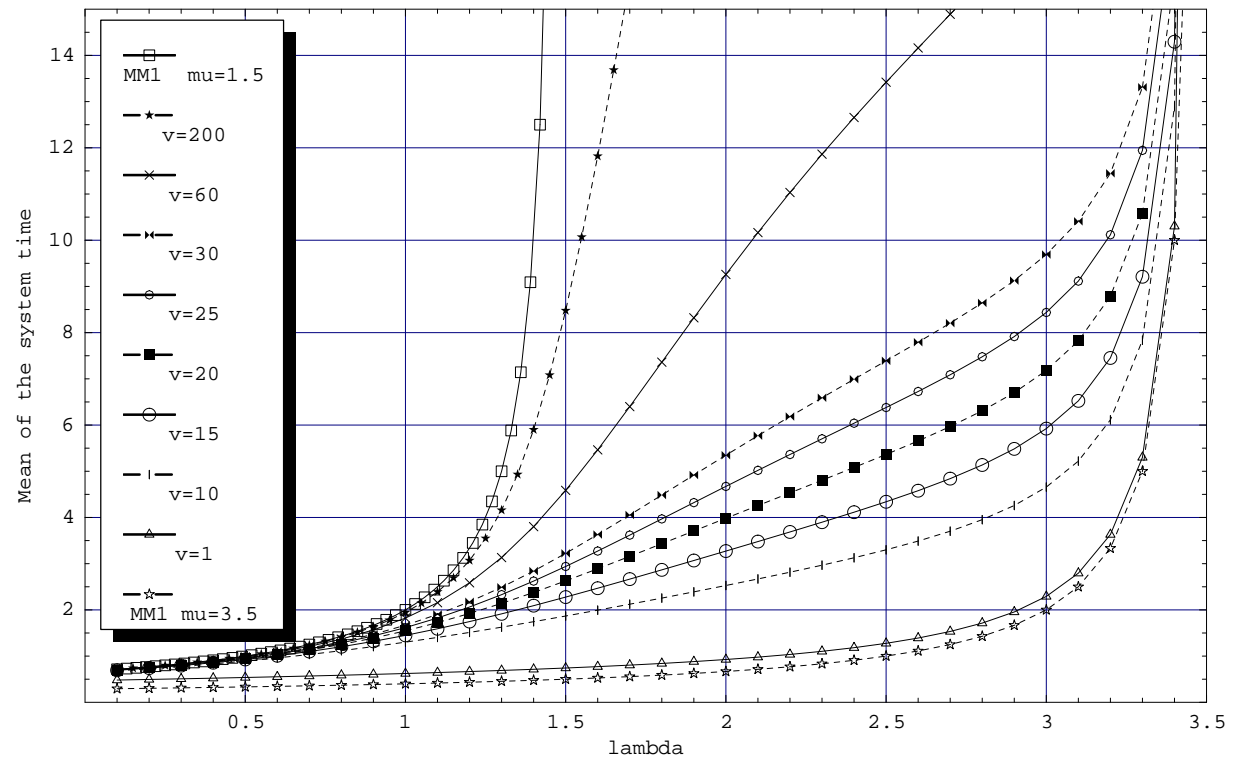

Figure 8: Mean of the system time when the service times in a working vacation and in a service period are exponentially distributed with $\nu=1.5, \mu=3.5$, respectively, and the length of a vacation is a constant $v$. MM1 means an M/M/1 queue.

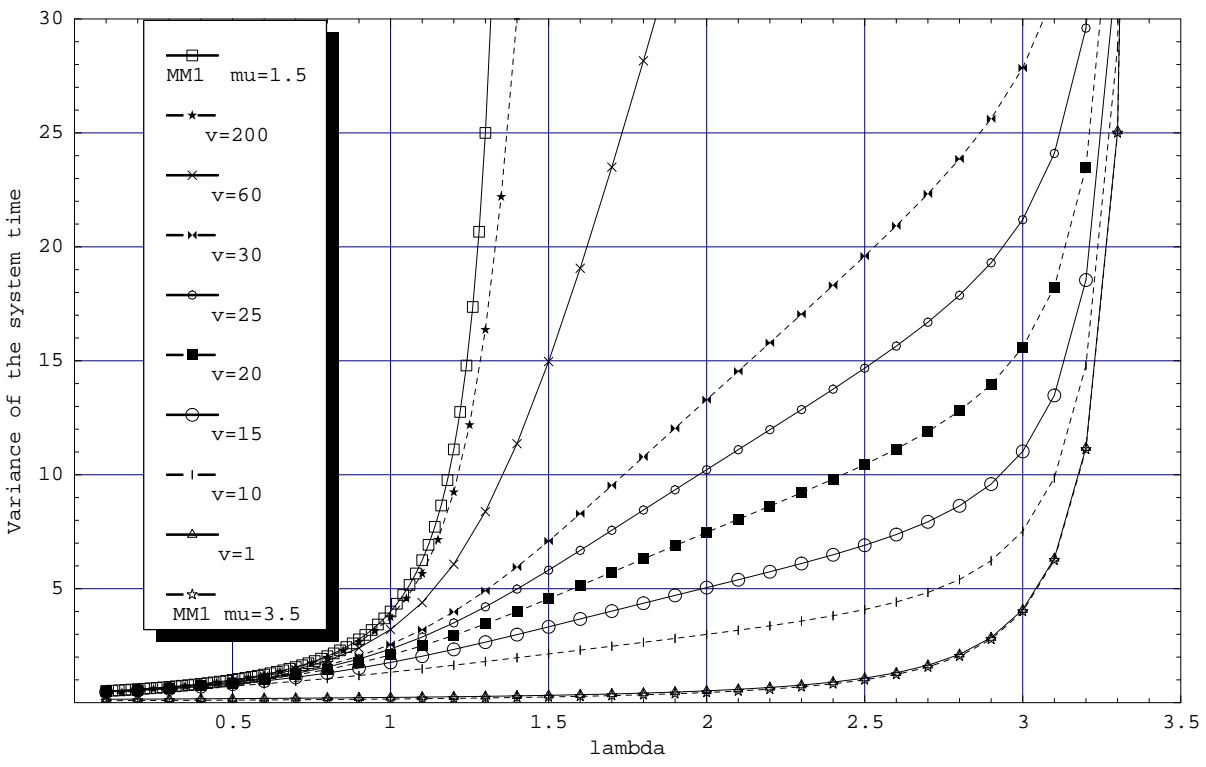

Figure 9: Variance of the system time when the service times in a working vacation and in a service period are exponentially distributed with $\nu=1.5, \mu=3.5$, respectively, and the length of a vacation is a constant $v$. MM1 means an $\mathrm{M} / \mathrm{M} / 1$ queue. 
and its LST is given by

$$
\phi(s)=\left(\frac{2 / v}{s+2 / v}\right)^{2} .
$$

As we stated earlier, $E_{n}$ in (69) can be calculated by directly applying the residue theorem. Thus we have

$$
\begin{aligned}
E_{n}= & \frac{1}{2 \pi \mathbf{i}} \int_{B r} \frac{\tau_{2}(s)}{s^{n} \nu\left[1-\tau_{2}(s)\right]} \phi(-s) d s=\left.\frac{-4}{\nu v^{2}} \frac{d}{d s}\left(\frac{\tau_{2}(s)}{s^{n}\left[1-\tau_{2}(s)\right]}\right)\right|_{s=\frac{2}{v}} \\
& =\frac{v^{n-2}}{2^{n+1} \nu}\left[(n+1)(\nu-\lambda) v^{2}-2 n v\right. \\
& \left.+\frac{(2+\lambda v)(\lambda v+2 n+\lambda n v)+\nu v[2-2 \lambda v+n(4-2 \lambda v)+(n+1) \nu v]}{\sqrt{(2 / v+\lambda+\nu)^{2}-4 \lambda \nu}}\right] .
\end{aligned}
$$

From (85), it is easy to verify that the mean of $V(t)$ is $v, v_{2}=(3 / 2) v$, and $v_{3}=3 v^{3}$.

Figure 10 and Figure 11 show the mean and variance, respectively, for the queue size. Figure 12 and Figure 13 show the mean and variance, respectively, for the system time. For comparison, the mean and variance of the queue size and the system time are also plotted in the corresponding figures. It is observed that the most features in this case are the same as in Example 1 except that the variances for the queue size and for the system time increase very quickly as $\lambda$ increases.

\section{Summary}

In this paper, we have analyzed an $\mathrm{M} / \mathrm{G} / 1 / \mathrm{WV}$ queue. The PGF for the queue size and the LST of the distribution function for the system time of an arbitrary customer have been derived by utilizing the transient solution for the queue size in an $M / G / 1$ queue. Two special cases are examined, i.e., the case when the length of the vacation follows an exponential distribution and the case in which the service time in a vacation is exponentially distributed. Several numerical examples are presented. It is observed that when the vacation length is very large or very small an $\mathrm{M} / \mathrm{G} / 1 / \mathrm{WV}$ queue behaves like an $\mathrm{M} / \mathrm{G} / 1$ queue. It is also observed that the variance of the length of a vacation influences strongly the variances of both queue size and system time.

\section{References}

[1] J. Abate, G. L. Choudhury, and W. Whitt, "An introduction to numerical transform inversion and its application to probability models," Computational Probability, W. K. Grassmann (editor), pp.257-323, Kluwer Academic Publishers, 2000.

[2] B. T. Doshi, "Queueing systems with vacations - a survey," Queueing Systems, Vol.1, No.1, pp. 29-66, June 1986. 


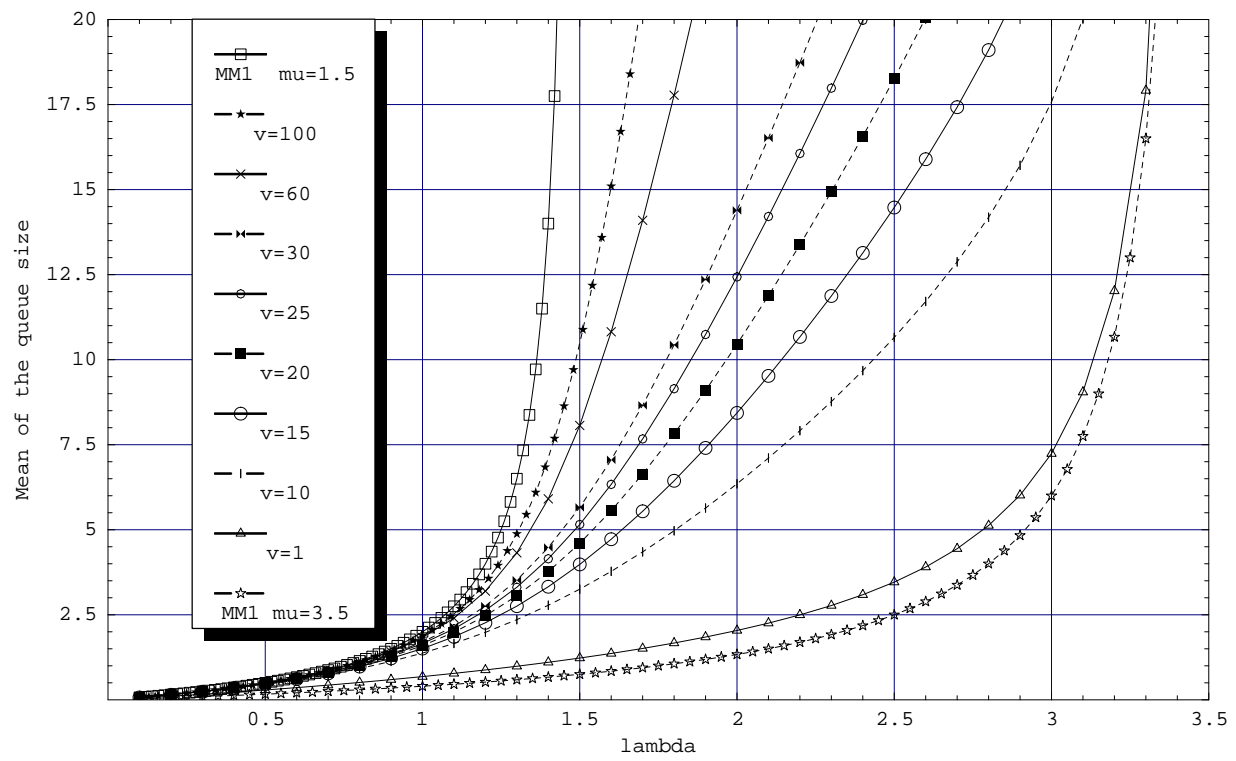

Figure 10: Mean of the queue size when the service times in a working vacation and in a service period are exponentially distributed with $\nu=1.5, \mu=3.5$, respectively, and the length of a vacation follows an Erlang distribution with order 2 and mean $v$. MM1 means an $\mathrm{M} / \mathrm{M} / 1$ queue.

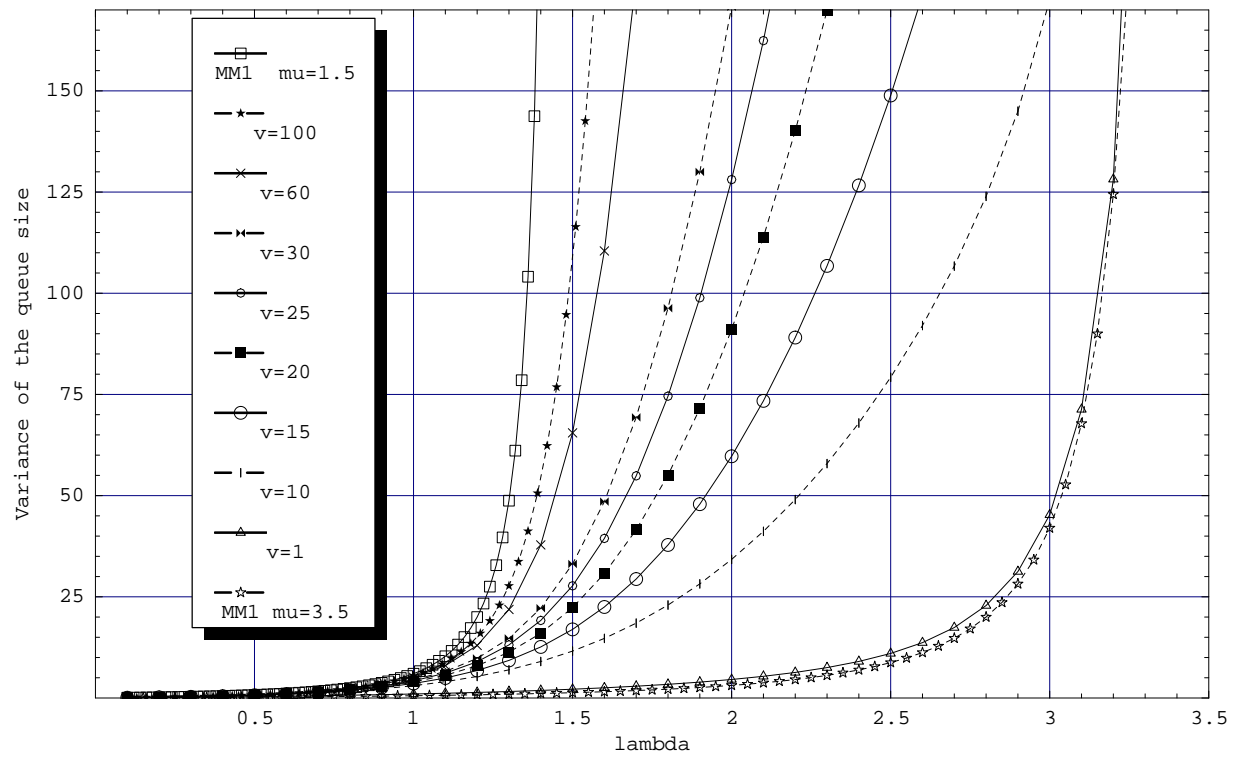

Figure 11: Variance of the queue size when the service times in a working vacation and in a service period are exponentially distributed with $\nu=1.5, \mu=3.5$, respectively, and the length of a vacation follows an Erlang distribution with order 2 and mean $v$. MM1 means an $\mathrm{M} / \mathrm{M} / 1$ queue. 


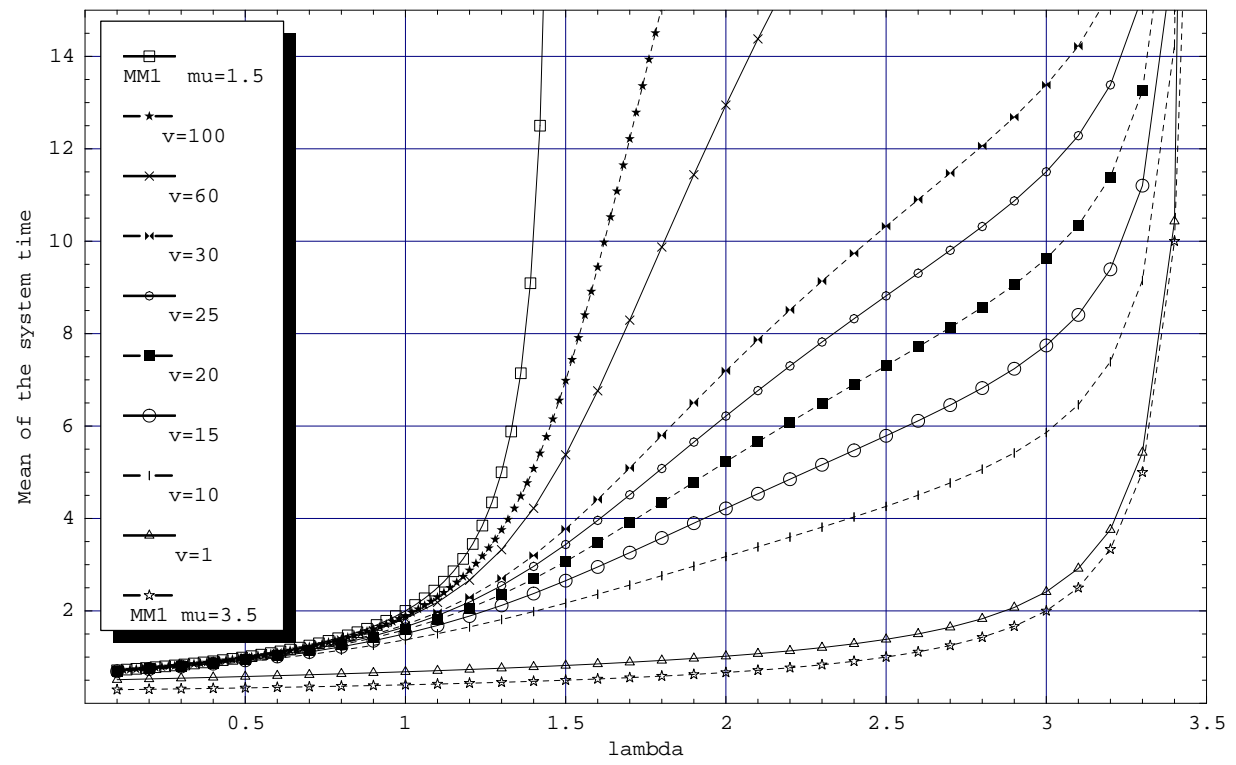

Figure 12: Mean of the system time when the service times in a working vacation and in a service period are exponentially distributed with $\nu=1.5, \mu=3.5$, respectively, and the length of a vacation follows an Erlang distribution with order 2 and mean $v$. MM1 means an $\mathrm{M} / \mathrm{M} / 1$ queue.

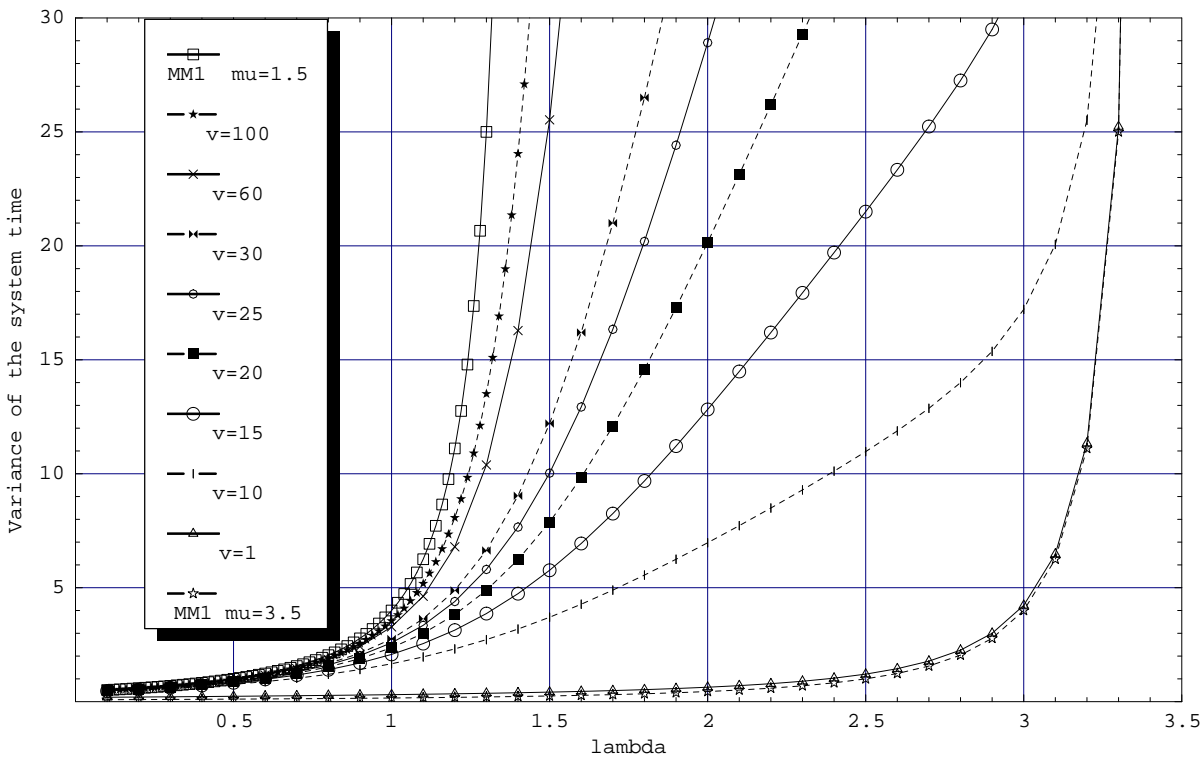

Figure 13: Variance of the system time when the service times in a working vacation and in the service period are exponentially distributed with $\nu=1.5, \mu=3.5$, respectively, and the length of a vacation follows an Erlang distribution with order 2 and mean $v$. MM1 means an M/M/1 queue. 
[3] B. T. Doshi, "Single server queues with vacations," Stochastic Analysis of Computer and Communication Systems, H. Takagi (editor), pp.217-226, North-Holland, Amsterdam, 1991.

[4] J. Keilson and L. D. Servi, "A distributional form of Little's law," Operations Research Letters, Vol.7, No.5, pp.223-227, October 1988.

[5] A. Kuczura, "Queues with mixed renewal and Poisson inputs," The Bell System Technical Journal, Vol.51, No.6, pp.1305-1326, July-August 1972.

[6] A. Kuczura, "Piecewise Markov processes," SIAM Journal on Applied Mathematics, Vol.24, No.2, pp.169-181, March 1973.

[7] A. Narula-Tam, S. G. Finn, and M. Medard, "Analysis of reconfiguration in IP over WDM access networks," Proceedings of the Optical Fiber Communication Conference (OFC), pp. MN4.1-MN4.3, 2001.

[8] L. D. Servi and S. G. Finn, "M/M/1 queues with working vacations (M/M/WV)," To appear in Performance Evaluation.

[9] L. Takács, Introduction to the Theory of Queues, Oxford University Press, New York, 1962.

[10] H. Takagi, Queueing Analysis: A Foundation of Performance Evaluation, Volume 1: Vacation and Priority Systems, Part 1, Elsevier, 1991.

\section{Appendices}

\section{A. Derivation of (42)}

If the server does not work during a vacation, it follows that $\psi(s)=0, \tau(s)=0$, and $N=0$. The PGF in (31) becomes

$$
\begin{aligned}
\hat{\Psi}(z) & =\frac{1}{2 \pi \mathbf{i}} \int_{B r} \frac{\hat{\phi}(-s)}{s+\lambda-\lambda z} d s=\frac{1}{v} \int_{0}^{\infty}(1-V(t)) d t \frac{1}{2 \pi \mathbf{i}} \int_{B r} \frac{e^{s t}}{s+\lambda-\lambda z} d s \\
& =\frac{1}{v} \int_{0}^{\infty}(1-V(t)) e^{-\lambda(1-z) t} d t=\frac{1-\phi(\lambda-\lambda z)}{\lambda v(1-z)} .
\end{aligned}
$$

Similarly, (12) can be written as

$$
\Psi(z)=\frac{1}{2 \pi \mathbf{i}} \int_{B r} \frac{\phi(-s)}{s+\lambda-\lambda z} d s=\phi(\lambda-\lambda z) .
$$

Substituting (A.1) and (A.2) into (34), we have

$$
\begin{aligned}
\Phi(z) & =\frac{1-\lambda b}{v}\left(\frac{1-\phi(\lambda-\lambda z)}{\lambda(1-z)}+\frac{z[\phi(\lambda-\lambda z)-1][1-\beta(\lambda-\lambda z)]}{\lambda(1-z)[z-\beta(\lambda-\lambda z)]}\right) \\
& =\frac{(1-\lambda b)[1-\phi(\lambda-\lambda z)] \beta(\lambda-\lambda z)}{\lambda v[\beta(\lambda-\lambda z)-z]},
\end{aligned}
$$

which is (42). 


\section{B. Derivation of (55)}

If the server is always on a vacation, we take the limit $\eta \rightarrow 0$ in (47). Since $\psi(\eta)=$ $1+O(\eta)$ and $\tau(\eta)=1+O(\eta)$ in this limit, we have

$$
\lim _{\eta \rightarrow 0} \frac{\eta(1-\lambda b)[1-\psi(\eta)]\{\eta+\lambda[1-\tau(\eta)]\}}{[1-\psi(\eta)]\{\eta+\lambda[1-\tau(\eta)]\}-b \lambda \eta[1-\tau(\eta)] \psi(\eta)}=0
$$

and

$$
\begin{aligned}
& \lim _{\eta \rightarrow 0} \frac{\eta(1-\lambda b)[1-\psi(\eta)]\{\eta+\lambda[1-\tau(\eta)]\}}{[1-\psi(\eta)]\{\eta+\lambda[1-\tau(\eta)]\}-b \lambda \eta[1-\tau(\eta)] \psi(\eta)} \Omega(z) \\
& =\lim _{\eta \rightarrow 0} \frac{\eta(1-\lambda b)[1-\psi(\eta)]}{[1-\psi(\eta)]\{\eta+\lambda[1-\tau(\eta)]\}-\lambda \eta b[1-\tau(\eta)] \psi(\eta)} \\
& \times \frac{z[1-\psi(\eta+\lambda-\lambda z)]\{\eta+\lambda[1-\tau(\eta)]\}+(z-1) \psi(\eta+\lambda-\lambda z)[\eta+\lambda(1-z)]}{[\eta+\lambda(1-z)][z-\psi(\eta+\lambda-\lambda z)]} \\
& =\lim _{\eta \rightarrow 0} \frac{(1-\lambda b)}{1+\frac{\lambda[1-\tau(\eta)]}{\eta}-\frac{\lambda b[1-\tau(\eta)]}{1-\psi(\eta)} \psi(\eta)} \\
& \times \frac{z[1-\psi(\eta+\lambda-\lambda z)]\{\eta+\lambda[1-\tau(\eta)]\}+(z-1) \psi(\eta+\lambda-\lambda z)[\eta+\lambda(1-z)]}{[\eta+\lambda(1-z)][z-\psi(\eta+\lambda-\lambda z)]} \\
& =\frac{(1-\lambda b)(z-1) \psi(\lambda-\lambda z) \lambda(1-z)}{\left(1+\frac{\lambda h}{1-\lambda h}-\frac{\lambda b}{1-\lambda h}\right) \lambda(1-z)[z-\psi(\lambda-\lambda z)]} \\
& =\frac{(1-\lambda h)(1-z) \psi(\lambda-\lambda z)}{\psi(\lambda-\lambda z)-z},
\end{aligned}
$$

where we have used

$$
\lim _{\eta \rightarrow 0} \frac{1-\tau(\eta)}{\eta}=\frac{h}{1-\lambda h}
$$

and

$$
\lim _{\eta \rightarrow 0} \frac{1-\tau(\eta)}{1-\psi(\eta)}=\frac{1}{1-\lambda h} .
$$

From (A.4) and (A.5), we also have

$$
\lim _{\eta \rightarrow 0} \frac{\eta(1-\lambda b)[1-\psi(\eta)]\{\eta+\lambda[1-\tau(\eta)]\}}{[1-\psi(\eta)]\{\eta+\lambda[1-\tau(\eta)]\}-b \lambda \eta[1-\tau(\eta)] \psi(\eta)}(\eta \Omega(z)-1)=0 .
$$

Using (A.5) and (A.6) in (47) yields

$$
\Phi(z)=\frac{(1-\lambda h)(1-z) \psi(\lambda-\lambda z)}{\psi(\lambda-\lambda z)-z},
$$

which is (55). 


\section{Derivation of (56)}

If the server never takes a vacation, we take the limit $\eta \rightarrow \infty$ in (47). In this case we have $\psi(\infty)=0, \tau(\infty)=0$, and

$$
\begin{aligned}
& \lim _{\eta \rightarrow \infty} \frac{\eta(1-\lambda b)[1-\psi(\eta)]\{\eta+\lambda[1-\tau(\eta)]\}}{[1-\psi(\eta)]\{\eta+\lambda[1-\tau(\eta)]\}-b \lambda \eta[1-\tau(\eta)] \psi(\eta)} \Omega(z) \\
& =\lim _{\eta \rightarrow \infty} \frac{\eta(1-\lambda b)[1-\psi(\eta)]}{[1-\psi(\eta)]\{\eta+\lambda[1-\tau(\eta)]\}-\lambda \eta b[1-\tau(\eta)] \psi(\eta)} \\
& \times \frac{z[1-\psi(\eta+\lambda-\lambda z)]\{\eta+\lambda[1-\tau(\eta)]\}+(z-1) \psi(\eta+\lambda-\lambda z)[\eta+\lambda(1-z)]}{[\eta+\lambda(1-z)][z-\psi(\eta+\lambda-\lambda z)]} \\
& =\lim _{\eta \rightarrow \infty} \frac{(1-\lambda b)[1-\psi(\eta)]}{[1-\psi(\eta)]\left(1+\frac{\lambda[1-\tau(\eta)]}{\eta}\right)-\lambda b[1-\tau(\eta)] \psi(\eta)} \\
& \\
& \times \frac{z[1-\psi(\eta+\lambda-\lambda z)]\left(1+\frac{\lambda[1-\tau(\eta)]}{\eta}\right)+(z-1) \psi(\eta+\lambda-\lambda z)\left(1+\frac{\lambda(1-z)}{\eta}\right)}{\left(1+\frac{\lambda(1-z)}{\eta}\right)[z-\psi(\eta+\lambda-\lambda z)]} \\
& =1-\lambda b .
\end{aligned}
$$

Since

$$
\begin{aligned}
& \eta \Omega(z)-1=\frac{1-z}{z-\psi(\eta+\lambda-\lambda z)} \\
& \times \frac{\{\eta+\lambda[1-\tau(\eta)]\}[\psi(\eta+\lambda-\lambda z)(\lambda+\eta)-\lambda z]-\psi(\eta+\lambda-\lambda z) \eta[\eta+\lambda(1-z)]}{[\eta+\lambda(1-z)]\{\eta+\lambda[1-\tau(\eta)]\}}
\end{aligned}
$$

it follows that

$$
\begin{aligned}
& \lim _{\eta \rightarrow \infty} \frac{\eta(1-\lambda b)[1-\psi(\eta)]\{\eta+\lambda[1-\tau(\eta)]\}}{[1-\psi(\eta)]\{\eta+\lambda[1-\tau(\eta)]\}-b \lambda \eta[1-\tau(\eta)] \psi(\eta)}[\eta \Omega(z)-1] \\
= & \lim _{\eta \rightarrow \infty} \frac{\eta(1-\lambda b)[1-\psi(\eta)](1-z)}{[\eta+\lambda(1-z)][z-\psi(\eta+\lambda-\lambda z)]} \\
& \times \frac{\{\eta+\lambda[1-\tau(\eta)]\}[\psi(\eta+\lambda-\lambda z)(\lambda+\eta)-\lambda z]-\psi(\eta+\lambda-\lambda z) \eta[\eta+\lambda(1-z)]}{[1-\psi(\eta)]\{\eta+\lambda[1-\tau(\eta)]\}-\lambda \eta b[1-\tau(\eta)] \psi(\eta)} \\
= & \lim _{\eta \rightarrow \infty} \frac{\lambda \eta(1-\lambda b)[1-\psi(\eta)](1-z)}{[\eta+\lambda(1-z)][z-\psi(\eta+\lambda-\lambda z)]} \\
& \times \frac{-z(\lambda+\eta)+[(z+1) \eta+\lambda] \psi(\eta+\lambda-\lambda z)+\tau(\eta)[z \lambda-(\eta+\lambda) \psi(\eta+\lambda-\lambda z)]}{[1-\psi(\eta)]\{\eta+\lambda[1-\tau(\eta)]\}-\lambda \eta b[1-\tau(\eta)] \psi(\eta)} \\
= & \lim _{\eta \rightarrow \infty} \frac{\lambda(1-\lambda b)[1-\psi(\eta)](1-z)}{\left(1+\frac{\lambda(1-z)}{\eta}\right)[z-\psi(\eta+\lambda-\lambda z)]} \\
& \times \frac{-z\left(1+\frac{\lambda}{\eta}\right)+\left((z+1)+\frac{\lambda}{\eta}\right) \psi(\eta+\lambda-\lambda z)+\tau(\eta)\left[\frac{z \lambda}{\eta}-\left(1+\frac{\lambda}{\eta}\right) \psi(\eta+\lambda-\lambda z)\right]}{[1-\psi(\eta)]\left(1+\frac{\lambda[1-\tau(\eta)]}{\eta}\right)-\lambda b[1-\tau(\eta)] \psi(\eta)}
\end{aligned}
$$




$$
=(z-1) \lambda(1-\lambda b)
$$

Substituting (A.7) and (A.9) into (47), we obtain

$$
\Phi(z)=\frac{(1-\lambda b)(1-z) \beta(\lambda-\lambda z)}{\beta(\lambda-\lambda z)-z},
$$

which is (56).

\section{Derivation of (83)}

Suppose that the length of a vacation follows an exponential distribution with mean $v=1 / \eta$ and that the service times in a vacation and in a service period are exponentially distributed with rate $\nu$ and $\mu=1 / b$, respectively. We consider $\Phi(z)$ in (70). First, substituting $\phi(s)=\eta /(s+\eta)$ and $\psi(s)=\nu /(s+\nu)$ into (15), we have

$$
\begin{aligned}
N & =\frac{1}{2 \pi \mathbf{i}} \int_{B r} \frac{\psi(s)}{s[1-\psi(s)]}\left(1-\frac{s}{s+\lambda\left(1-\tau_{2}(s)\right)}\right) \phi(-s) d s \\
& =\frac{\lambda \psi(\eta)\left[1-\tau_{2}(\eta)\right]}{[1-\psi(\eta)]\left\{\eta+\lambda\left[1-\tau_{2}(\eta)\right]\right\}}=\frac{\lambda \nu\left[1-\tau_{2}(\eta)\right]}{\eta\left\{\eta+\lambda\left[1-\tau_{2}(\eta)\right]\right\}} .
\end{aligned}
$$

From $(3), \tau_{1}(\eta)$ and $\tau_{2}(\eta)$ are the roots of the quadratic

$$
\lambda z^{2}-(\eta+\lambda+\nu) z+\nu=0
$$

Thus we have

$$
\eta=\lambda\left[\tau_{1}(\eta)+\tau_{2}(\eta)\right]-\nu-\lambda
$$

and

$$
\tau_{2}(\eta)=\frac{\nu}{\lambda \tau_{1}(\eta)}
$$

It follows that

$$
\begin{aligned}
& \frac{1-\lambda b}{v-b N} \\
= & \frac{\eta(\mu-\lambda)\left\{\eta+\lambda\left[1-\tau_{2}(\eta)\right]\right\}}{\mu\left\{\eta+\lambda\left[1-\tau_{2}(\eta)\right]\right\}-\lambda \nu\left[1-\tau_{2}(\eta)\right]} \\
= & \frac{(\mu-\lambda)\left\{\lambda\left[\tau_{1}(\eta)+\tau_{2}(\eta)\right]-\nu-\lambda\right\}\left\{\lambda\left[\tau_{1}(\eta)+\tau_{2}(\eta)\right]-\nu-\lambda+\lambda\left[1-\tau_{2}(\eta)\right]\right\}}{\mu\left\{\lambda\left[\tau_{1}(\eta)+\tau_{2}(\eta)\right]-\nu-\lambda+\lambda\left[1-\tau_{2}(\eta)\right]\right\}-\lambda \nu\left[1-\tau_{2}(\eta)\right]} \\
= & \frac{(\mu-\lambda)\left\{\lambda\left[\tau_{2}(\eta)-1\right]+\lambda \tau_{1}(\eta)-\nu\right\}\left[\lambda \tau_{1}(\eta)-\nu\right]}{\mu\left[\lambda \tau_{1}(\eta)-\nu\right]-\lambda \nu\left[1-\tau_{2}(\eta)\right]} \\
= & \frac{(\mu-\lambda)\left\{\lambda \frac{\nu-\lambda \tau_{1}(\eta)}{\lambda \tau_{1}(\eta)}-\left[\nu-\lambda \tau_{1}(\eta)\right]\right\}\left[\lambda \tau_{1}(\eta)-\nu\right]}{\mu\left[\lambda \tau_{1}(\eta)-\nu\right]-\lambda \nu \frac{\lambda \tau_{1}(\eta)-\nu}{\lambda \tau_{1}(\eta)}} \\
= & \frac{(\mu-\lambda)\left[\nu-\lambda \tau_{1}(\eta)\right]^{2}\left[\tau_{1}(\eta)-1\right] / \tau_{1}(\eta)}{\left[\lambda \tau_{1}(\eta)-\nu\right]\left[\mu \tau_{1}(\eta)-\nu\right] / \tau_{1}(\eta)}
\end{aligned}
$$




$$
=\frac{(\mu-\lambda)\left[\lambda \tau_{1}(\eta)-\nu\right]\left[\tau_{1}(\eta)-1\right]}{\mu \tau_{1}(\eta)-\nu} .
$$

From $\hat{\Psi}(z)$ in (72) we obtain

$$
\begin{aligned}
v \hat{\Psi}(z) & =v \frac{1}{2 \pi \mathbf{i}} \int_{B r}\left[\frac{z+(z-1) \frac{\tau_{2}(s)}{1-\tau_{2}(s)}}{z s-(1-z)(\nu-\lambda z)}\right] \hat{\phi}(-s) d s \\
& =\frac{z+(z-1) \frac{\tau_{2}(\eta)}{1-\tau_{2}(\eta)}}{z \eta-(1-z)(\nu-\lambda z)} \\
& =\frac{z+(z-1) \frac{\nu}{\lambda \tau_{1}(\eta)-\nu}}{\frac{z\left[1-\tau_{1}(\eta)\right]\left[\nu-\lambda \tau_{1}(\eta)\right]}{\tau_{1}(\eta)}-(1-z)(\nu-\lambda z)} \\
& =\frac{\left[\lambda \tau_{1}(\eta) z-\nu\right] \tau_{1}(\eta)}{\left[\lambda \tau_{1}(\eta)-\nu\right]\left[z \nu+\lambda \tau_{1}(\eta)^{2} z-\nu \tau_{1}(\eta)-\lambda \tau_{1}(\eta) z^{2}\right]} \\
& =\frac{-\tau_{1}(\eta)\left[\lambda \tau_{1}(\eta) z-\nu\right]}{\left[\lambda \tau_{1}(\eta)-\nu\right]\left[z-\tau_{1}(\eta)\right]\left[\lambda \tau_{1}(\eta) z-\nu\right]} \\
& =\frac{-\tau_{1}(\eta)}{\left[\lambda \tau_{1}(\eta)-\nu\right]\left[z-\tau_{1}(\eta)\right]}
\end{aligned}
$$

where we have used

$$
\frac{\tau_{2}(\eta)}{1-\tau_{2}(\eta)}=\frac{\frac{\nu}{\lambda \tau_{1}(\eta)}}{1-\frac{\nu}{\lambda \tau_{1}(\eta)}}=\frac{\nu}{\lambda \tau_{1}(\eta)-\nu}
$$

and

$$
\begin{aligned}
\eta & =\lambda\left[\tau_{1}(\eta)+\tau_{2}(\eta)\right]-\nu-\lambda=\lambda\left[\tau_{1}(\eta)+\frac{\nu}{\lambda \tau_{1}(\eta)}\right]-\nu-\lambda \\
& \left.=\lambda\left[\tau_{1}(\eta)-1\right)\right]+\nu\left[\frac{1}{\tau_{1}(\eta)}-1\right]=\frac{\left[1-\tau_{1}(\eta)\right]\left[\nu-\lambda \tau_{1}(\eta)\right]}{\tau_{1}(\eta)} .
\end{aligned}
$$

Using (A.15) and (A.17) in (71) yields

$$
\begin{aligned}
\Psi(z) & =\frac{1}{2 \pi \mathbf{i}} \int_{B r}\left[\frac{z+(z-1) \frac{\tau_{2}(s)}{1-\tau_{2}(s)}}{z s-(1-z)(\nu-\lambda z)}\right] \phi(-s) d s \\
& =\eta \frac{z+(z-1) \frac{\tau_{2}(\eta)}{1-\tau_{2}(\eta)}}{z \eta-(1-z)(\nu-\lambda z)} \\
& =\left(\frac{\left[1-\tau_{1}(\eta)\right]\left[\nu-\lambda \tau_{1}(\eta)\right]}{\tau_{1}(\eta)}\right)\left(\frac{-\tau_{1}(\eta)}{\left[\lambda \tau_{1}(\eta)-\nu\right]\left[z-\tau_{1}(\eta)\right]}\right) \\
& =\frac{1-\tau_{1}(\eta)}{z-\tau_{1}(\eta)} .
\end{aligned}
$$

It is easy to verify that

$$
\frac{z[1-\beta(\lambda-\lambda z)]}{\lambda(1-z)[z-\beta(\lambda-\lambda z)]}=\frac{z\left[1-\frac{\mu}{\mu+\lambda(1-z)}\right]}{\lambda(1-z)\left[z-\frac{\mu}{\mu+\lambda(1-z)}\right]}=\frac{z}{(1-z)(\lambda z-\mu)} .
$$


Substituting (A.14), (A.15), (A.18) and (A.19) into (70) we obtain

$$
\begin{aligned}
& \Phi(z) \\
= & \frac{(\mu-\lambda)\left[\lambda \tau_{1}(\eta)-\nu\right]\left[\tau_{1}(\eta)-1\right]}{\mu \tau_{1}(\eta)-\nu}\left(\frac{-\tau_{1}(\eta)}{\left[\lambda \tau_{1}(\eta)-\nu\right]\left[z-\tau_{1}(\eta)\right]}+\frac{z}{\left[z-\tau_{1}(\eta)\right](\lambda z-\mu)}\right) \\
= & \frac{(\mu-\lambda)\left[\lambda \tau_{1}(\eta)-\nu\right]\left[\tau_{1}(\eta)-1\right]}{\mu \tau_{1}(\eta)-\nu}\left(\frac{\mu \tau_{1}(\eta)-\nu z}{(\mu-\lambda z)\left[\tau_{1}(\eta)-z\right]\left[\lambda \tau_{1}(\eta)-\nu\right]}\right) \\
= & \left(\frac{\mu-\lambda}{\mu-\lambda z}\right)\left(\frac{\tau_{1}(\eta)-1}{\tau_{1}(\eta)-z}\right)\left(\frac{\mu \tau_{1}(\eta)-\nu}{\mu \tau_{1}(\eta)-z \nu}\right)^{-1} \\
= & \left(\frac{1-\frac{\lambda}{\mu}}{1-\frac{\lambda}{\mu} z}\right)\left(\frac{1-\tau_{1}(\eta)^{-1}}{1-\tau_{1}(\eta)^{-1} z}\right)\left(\frac{1-\frac{\nu}{\tau_{1}(\eta) \mu}}{1-\frac{\nu}{\tau_{1}(\eta) \mu} z}\right)^{-1},
\end{aligned}
$$

which is (83). 